OPEN ACCESS

Edited by:

Jonathan Shaw,

University of Sheffield, UK

Reviewed by:

Yajun Song,

Beijing Institute of Microbiology and

Epidemiology, China

Kai Zhou,

Zhejiang University, China

*Correspondence:

Joseph C. Newton

newtojc@auburn.edu

Mark R. Liles

lilesma@auburn.edu

Specialty section:

This article was submitted to

Infectious Diseases,

a section of the journal

Frontiers in Microbiology

Received: 30 June 2016 Accepted: 27 September 2016

Published: 18 October 2016

Citation:

Rasmussen-Ivey CR, Hossain MJ,

Odom SE, Terhune JS,

Hemstreet WG, Shoemaker $C A$,

Zhang D, Xu D-H, Griffin MJ, Liu Y-J,

Figueras MJ, Santos SR, Newton JC

and Liles MR (2016) Classification of a

Hypervirulent Aeromonas hydrophila

Pathotype Responsible for Epidemic

Outbreaks in Warm-Water Fishes.

Front. Microbiol. 7:1615.

doi: 10.3389/fmicb.2016.01615

\section{Classification of a Hypervirulent Aeromonas hydrophila Pathotype Responsible for Epidemic Outbreaks in Warm-Water Fishes}

Cody R. Rasmussen-Ivey ${ }^{1}$, Mohammad J. Hossain ${ }^{1}$, Sara E. Odom ${ }^{1}$, Jeffery S. Terhune ${ }^{2}$, William G. Hemstreet ${ }^{3}$, Craig A. Shoemaker ${ }^{4}$, Dunhua Zhang ${ }^{4}$, De-Hai Xu ${ }^{4}$, Matt J. Griffin ${ }^{5}$, Yong-Jie Liu ${ }^{6}$, Maria J. Figueras ${ }^{7}$, Scott R. Santos ${ }^{1}$, Joseph C. Newton ${ }^{8 *}$ and Mark R. Liles ${ }^{1 *}$

\footnotetext{
'Department of Biological Sciences, Auburn University, Auburn, AL, USA, ${ }^{2}$ School of Fisheries, Aquaculture and Aquatic Sciences, Auburn, AL, USA, ${ }^{3}$ Alabama Fish Farming Center, Greensboro, AL, USA, ${ }^{4}$ Aquatic Animal Health Research Unit, United States Department of Agriculture-Agricultural Research Service, Auburn, AL, USA, ${ }^{5}$ Thad Cochran National Warmwater Aquaculture Center, College of Veterinary Medicine, Mississippi State University, Stoneville, MS, USA, ${ }^{6}$ College of Veterinary Medicine, Nanjing Agricultural University, Nanjing, China, ${ }^{7}$ Departamento de Ciencias Médicas Básicas, Facultad de Medicina y Ciencias de la Salud, IISPV, Universidad Rovira i Virgili, Reus, Spain, ${ }^{8}$ Department of Pathobiology, Auburn University, Auburn, AL, USA
}

Lineages of hypervirulent Aeromonas hydrophila (vAh) are the cause of persistent outbreaks of motile Aeromonas septicemia in warm-water fishes worldwide. Over the last decade, this virulent lineage of $A$. hydrophila has resulted in annual losses of millions of tons of farmed carp and catfish in the People's Republic of China and the United States (US). Multiple lines of evidence indicate US catfish and Asian carp isolates of A. hydrophila affiliated with sequence type 251 (ST251) share a recent common ancestor. To address the genomic context for the putative intercontinental transfer and subsequent geographic spread of this pathogen, we conducted a core genome phylogenetic analysis on 61 Aeromonas spp. genomes, of which 40 were affiliated with $A$. hydrophila, with 26 identified as epidemic strains. Phylogenetic analyses indicate all ST251 strains form a coherent lineage affiliated with $A$. hydrophila. Within this lineage, conserved genetic loci unique to $A$. hydrophila were identified, with some genes present in consistently higher copy numbers than in non-epidemic $A$. hydrophila isolates. In addition, results from analyses of representative ST251 isolates support the conclusion that multiple lineages are present within US vAh isolated from Mississippi, whereas vAh isolated from Alabama appear clonal. This is the first report of genomic heterogeneity within US vAh isolates, with some Mississippi isolates showing closer affiliation with the Asian grass carp isolate ZC1 than other vAh isolated in the US. To evaluate the biological significance of the identified heterogeneity, comparative disease challenges were conducted with representatives of different vAh genotypes. These studies revealed that isolate ZC1 yielded significantly lower mortality in channel catfish, relative to Alabama and Mississippi vAh isolates. Like other Asian vAh isolates, the ZC1 lineage contains all core genes for a complete type VI 
secretion system (T6SS). In contrast, more virulent US isolates retain only remnants of the T6SS (c/pB, hcp, vgrG, and vasH) which may have functional implications. Collectively, these results characterize a hypervirulent $A$. hydrophila pathotype that affects farmed fish on multiple continents.

Keywords: Aeromonas hydrophila, pathogenesis, comparative genomics, emerging disease, bacteria, catfish, carp

\section{INTRODUCTION}

Aquaculture industries across the world have been decimated by epidemics of a hypervirulent pathotype of $A$. hydrophila (vAh) (Nielsen et al., 2001; Hemstreet, 2010). A. hydrophila is ubiquitous within warm-water environments and has a diverse host range (i.e., amphibians, birds, fishes, reptiles, and mammals) with equally diverse diseases that include motile Aeromonas septicemia (MAS) in fish. MAS produces significant internal and external hemorrhage and exophthalmia, often followed by mortality within several hours of manifestation of disease (Xu et al., 1993; Camus et al., 1998; Nielsen et al., 2001; da Silva et al., 2012; Beaz-Hidalgo et al., 2013).

The first report of the vAh pathotype was A. hydrophila J-1 in 1989, which was isolated from epizootics of MAS in China's Jiangsu Province (Chen and Lu, 1991). This strain was categorized as sequence type 251 (ST251) and recognized as being capable of causing high mortality in grass carp (Ctenopharyngodon idella). Outbreaks of MAS in farmed carp have persisted in China, resulting in losses estimated at 2,200 tons of dead fish per year (Chen and Lu, 1991; Nielsen et al., 2001; Zhang et al., 2002; Pang et al., 2015). Epidemics of ST251associated MAS occurred within the same province in 2010, with vAh isolate $A$. hydrophila NJ-35 identified as the etiologic agent (Pang et al., 2012). Another vAh isolate from China (A. hydrophila ZC1) was isolated from grass carp exhibiting signs of hemorrhagic septicemia from an aquaculture farm in China's Guangdong Province (Deng et al., 2009). In general, MAS is a regular occurrence each summer, resulting in significant economic losses in the Chinese aquaculture industry, with estimates exceeding five billion yuan per year (Prof. Hui Chen, personal communication).

In 2004, the first reported case of ST251-related MAS in the US arose when A. hydrophila S04-690 was isolated from diseased channel catfish (Ictalurus punctatus) from a catfish farm in Washington County, Mississippi (MS) (Hossain et al., 2014). Beginning in 2009, vAh strains were consistently recovered from recurring outbreaks of MAS in aquaculture ponds in western Alabama (AL) with a reported 2,000 tons of dead fish in the first year (Hemstreet, 2010). To date, this number has grown to exceed an estimated 10,500 tons, with vAh isolates representing the largest percentage (35\%) of disease cases at the Alabama Fish Farming Center (Hemstreet, 2015). Although representative data on production losses attributed solely to vAh are difficult to attain, vAh clearly represents a significant threat to warm-water aquaculture industries.

Previous comparative genomic analyses of vAh strains isolated from catfish in the US and carp in China indicated these strains share a recent common ancestor (Hossain et al., 2014). Within this monophyletic clade, vAh strains isolated from carp and catfish have unique phenotypes and genotypes (L-fucose metabolism, an inducible prophage and the ability to use myoinositol as a sole carbon source) that distinguish them from more typical strains of $A$. hydrophila not associated with epizootics (non-vAh) (Hossain et al., 2013, 2014; Pang et al., 2015). These studies also demonstrated that MS vAh strain S04-690 is more similar to the carp isolate ZC1 than to other vAh strains from AL (Hossain et al., 2014). Since this study was published, MAS outbreaks attributed to vAh have spread to the Delta region of west Mississippi, with anecdotal reports from the industry suggesting annual losses in MS now exceed 150 tons. The purpose of this study was to characterize the vAh pathotype by examining vAh strains collected from farmed catfish in AL and MS in recent years and compare genome sequences of all available ST251 strains together with other Aeromonas spp. genomes available in GenBank by phylogenomic analysis, determine the presence of putative virulence factors in vAh and non-vAh lineages and assess the relative capacity of selected strains to cause MAS in channel catfish.

\section{METHODS}

\section{Bacterial Strains-Disease Isolates and Catfish Challenge}

A. hydrophila isolates for disease challenge were recovered from diseased channel catfish from commercial aquaculture operations in western Alabama and the Delta region of west Mississippi. Briefly, catfish demonstrating symptoms typical of MAS were collected in a moribund state and submitted for diagnostic evaluation and necropsy at Auburn University in Auburn, AL or Mississippi State University's Aquatic Research and Diagnostic Laboratory at the Thad Cochran National Warmwater Aquaculture Center (NWAC) in Stoneville, MS. Liver and kidney tissues were sampled for aerobic bacterial cultures. Samples of tissue were homogenized in sterile phosphate buffered saline and portions of the homogenate streaked onto tryptic soy agar (TSA; Beckton Dickinson, Franklin Lakes, NJ) or brain heart infusion (BHI; Beckton Dickinson) for bacterial isolation. Pure cultures were identified as vAh strains by the vAh-specific qPCR method previously described and/or utilization of myo-inositol as a sole carbon source (Griffin et al., 2013; Hanson et al., 2014). From this sampling design, A. hydrophila isolates ML09-119, ML10$51 \mathrm{~K}$, S04-690, S14-296, and S14-452 were cryogenically preserved (mixed with $50 \%$ glycerol, stored at $-80^{\circ} \mathrm{C}$ ) and subsequently used in catfish immersion challenges. 


\section{Infection and Histopathology of Channel Catfish Using an Immersion Challenge Model}

Channel catfish were obtained as fry from the USDA-ARS Warmwater Aquaculture Research Unit housed at NWAC and reared to experimental size in 340 liter troughs supplied with $26 \pm 2{ }^{\circ} \mathrm{C}$ dechlorinated municipal water under pathogen-free conditions. All animal experiments were approved by, and conducted in compliance with, regulations of the Institutional Animal Care and Use Committee of the Aquatic Animal Health Research Unit (USDA-ARS) in Auburn, Alabama. Water temperature was maintained at $26 \pm 2{ }^{\circ} \mathrm{C}$ with a centralized heater. Prior to trials, heart, liver, head kidney, trunk kidney, spleen, brain, and skeletal muscle tissues were collected from 10 randomly sampled fish and sampled by culturing on BHI to verify fish were not presently infected with vAh.

Two hundred catfish fingerlings, with a mean weight of $111 \pm$ $47 \mathrm{~g}$ and length of $19 \pm 3 \mathrm{~cm}$, were acclimated for 12 days in 56-L glass aquaria (10 fish per tank, 3 tanks per isolate, and 2 mock infected control tanks) containing about 50-L water prior to challenge. The immersion challenge was conducted using the recently described fin clip method (Zhang et al., 2016). At the time of infection, water volume was reduced to $15-\mathrm{L}$ per tank. To sedate animals for handling, fish were netted from individual aquaria and placed into a container filled with 20 -L of dechlorinated water containing $150 \mathrm{mg} / \mathrm{L}$ of buffered Tricaine$\mathrm{S}$ (tricaine methanesulfonate; Western Chemical, Inc., Ferndale, WA). Once fish were anesthetized, the adipose fin was clipped at its base and fish were returned to respective aquaria for recovery from anesthesia.

For the bacterial challenge, $100 \mathrm{~mL}$ of tryptic soy broth (TSB) containing approximately $3.0 \times 10^{9} \mathrm{CFU} / \mathrm{mL}$ of the respective A. hydrophila strains (ML-09-119, ML10-51K, S04-690, S14-296, S14-452, and ZC1) was added to each of three aquaria, resulting in an approximate challenge dose of $\sim 2.0 \times 10^{7} \mathrm{CFU} / \mathrm{mL}$. Two tanks served as mock infected controls, receiving only $100 \mathrm{~mL}$ sterile TSB. After 1-h exposure, water flow to aquaria $(0.5 \mathrm{~L} / \mathrm{min})$ was resumed. Fish mortality was monitored daily for 7 days. At least $50 \%$ of dead fish were sampled for confirmation for the presence of vAh in liver and kidney tissues using M9 minimal medium containing $0.3 \%$ (w/v) myo-inositol (M9I) agar (Hanson et al., 2014). Moribund fish were removed from aquaria daily and surviving fish were euthanized by at least $15 \mathrm{~min}$ exposure to $300 \mathrm{mg} / \mathrm{L}$ buffered Tricaine-S solution, then necropsied. Heart, liver, head kidney, trunk kidney, spleen, brain, and skeletal muscle tissues were harvested and fixed in $10 \%$ buffered formalin for histopathology. Tissues were also collected and used for bacterial identification and quantitation. Formalin fixed tissues were processed and embedded in paraffin. The tissues were cut in four micron sections, stained with hematoxylin and eosin and evaluated for microscopic lesions by light microscopy.

\section{Bacterial Strains-Comparative Genomics}

In total, 61 complete and draft Aeromonas spp. genomes were included in this study (Table 1) which included A. hydrophila representative genomes as well as Aeromonas spp. genomes that are now recognized as having had an erroneous affiliation with A. hydrophila (Figueras et al., 2014; Beaz-Hidalgo et al., 2015a). These genomes were retrieved from the US National Center for Biotechnology Information (NCBI) GenBank database and included two A. caviae isolates, one A. dhakensis isolate, one A. enteropelogenes isolate, one $A$. media isolate, one A. molluscorum isolate, one $A$. taiwanensis isolate, one Aeromonas sp. isolate, 26 A. hydrophila fish vAh disease isolates, and 28 non-vAh $A$. hydrophila isolates.

\section{Genome Sequencing}

Strains representative of different vAh lineages were selected for Illumina sequencing based on results of vAh genotypespecific PCR (see below) from a total of 38 suspected vAh isolates recovered from diagnostic cases in MS between 2013 and 2015. Strains were selected so that isolates represented MAS outbreaks from multiple geographically discrete operations. Genome sequencing with 250 bp read-length using paired-end sequencing was performed on the Illumina MiSeq platform using the Nextera XT kit (Illumina, San Diego, CA) to prepare bar-coded fragment libraries according to the manufacturer's protocol. Sequence reads were trimmed and quality sequence reads were assembled de novo using the CLC Genomics Workbench (Qiagen, Redwood City, CA) using default settings. vAh strain draft genomes $(n=14)$ were generated for strains Ahy_Idx7_1, ALG15-098, IPRS-15-28, ML10-51K, S13-612, S13700, S14-230, S14-296, S14-458, S14-606, S15-130, S15-242, S15400, and S15-591 (Supplemental Table 1). In addition to a standard Illumina MiSeq run for vAh strain S14-452, a NxSeq $20 \mathrm{~kb}$ mate pair library was constructed and sequenced using an Illumina MiSeq at the Lucigen Corporation (Middleton, $\mathrm{WI}$ ). The de novo assembly from the standard Illumina MiSeq sequences resulted in 13 contigs ( 80.15 average coverage) whereas the combination of these sequences together with the mate pairderived sequences using de novo assembly with SPAdes (v3.5.0) resulted in a complete genome sequence.

\section{Pathotype-Specific PCR for vAh Genotypes}

Evaluation of the previously described vAh-specific qPCR primer set (listed as 2986L and 2986R in Table 2) (Griffin et al., 2013) was performed in silico using Geneious v. R9 with a maximum mismatch setting of two bases and a band prediction interval of between 100 and 1000 bases, which predicted that numerous vAh isolates (Ahy_Idx7_1, J-1, AL09-79, AL10-121, JBN2301, ML09121, ML09-122, NJ-35, PB10-118, S13-612, and S15-591) would not produce an amplicon. To address this potential pitfall, we evaluated the primer set using touchdown PCR on all available and relevant vAh and non-vAh isolates (data not shown). Touchdown PCR was performed on an Eppendorf Mastercycler Gradient S with $50 \mathrm{ng}$ of template gDNA extracted from each isolate (E.Z.N.A. ${ }^{\circledR}$ Bacterial DNA Kit; Omega Biotek, Georgia, USA), $13 \mu \mathrm{l}$ of Econotaq Plus Green 2x MasterMix (Lucigen, Madison, Wisconsin, USA), and 20 picomoles of each primer in a $25-\mu 1$ reaction. Cycling parameters comprised of an initial denaturation of $94^{\circ} \mathrm{C}$ for $3 \mathrm{~min} ; 10 \mathrm{cycles}$ of $94^{\circ} \mathrm{C}$ for $30 \mathrm{~s}, 68^{\circ} \mathrm{C}$ for $30 \mathrm{~s}\left(-1^{\circ} \mathrm{C}\right.$ per cycle), and $72^{\circ} \mathrm{C}$ for $1 \mathrm{~min}$; followed by 25 cycles of $94^{\circ} \mathrm{C}$ for $30 \mathrm{~s}, 58^{\circ} \mathrm{C}$ for $30 \mathrm{~s}$, and $72^{\circ} \mathrm{C}$ for $1 \mathrm{~min}$; and a 
TABLE 1 | Bacterial genomes used in comparative genomic analyses.

\begin{tabular}{|c|c|c|c|c|c|c|}
\hline Strain & Phenotype & Isolation source & $\begin{array}{l}\text { GenBank species } \\
\text { assignation }\end{array}$ & $\begin{array}{l}\text { Species based on } \\
\text { phylogeny and ANI }\end{array}$ & Accession & Reference \\
\hline Ae398 & Non-vAh & Human & A. caviae & A. caviae & $\underline{\text { SAMEA2272404 }}$ & Beatson et al., 2011 \\
\hline AAK1 & Non-vAh & Clinical & A. dhakensis & A. dhakensis & $\underline{\text { SAMD00036618 }}$ & Martínez-Murcía et al., 2008 \\
\hline 1999lcr & Non-vAh & Clinical & A. trota & A. molluscorum & $\underline{\text { SAMN02732394 }}$ & Dallagassa et al., Unpublished \\
\hline 116 & Non-vAh & Clinical & A. hydrophila & A. dhakensis & NZ ANPN00000000.1 & Chan et al., 2011 \\
\hline 173 & Non-vAh & Clinical & A. hydrophila & A. dhakensis & $\underline{N Z \_A O B N 00000000.1}$ & Chan et al., 2011 \\
\hline 187 & Non-vAh & Clinical & A. hydrophila & A. dhakensis & $\underline{\text { NZ_AOBO00000000.1 }}$ & Chan et al., 2011 \\
\hline 226 & Non-vAh & Clinical & A. hydrophila & A. hydrophila & $\underline{\text { NZ_JEML00000000.1 }}$ & Chan et al., 2011 \\
\hline 259 & Non-vAh & Clinical & A. hydrophila & A. dhakensis & NZ_AOBP00000000.1 & Chan et al., 2011 \\
\hline 277 & Non-vAh & Clinical & A. hydrophila & A. dhakensis & NZ_AOBQ00000000.1 & Chan et al., 2011 \\
\hline AL06-01 & Non-vah & Bluegill & A. hydrophila & A. hydrophila & $\underline{\text { SAMN01085623 }}$ & Hossain et al., 2013 \\
\hline AL06-06 & Non-vAh & Goldfish & A. hydrophila & A. hydrophila & $\underline{\text { NZ_CP010947.1 }}$ & Tekedar et al., 2015 \\
\hline AL10-121 & Non-vAh & Channel catfish & A. hydrophila & A. hydrophila & NZ_LRRW00000000.1 & Hossain, 2012 \\
\hline AL97-91 & Non-vAh & Channel catfish & A. hydrophila & A. hydrophila & $\underline{\text { SAMN04967787 }}$ & Hossain, 2012 \\
\hline${\text { ATCC } 7966^{\top}}^{\top}$ & Non-vAh & Milk tin & A. hydrophila & A. hydrophila & $\underline{\text { NC_008570.1 }}$ & Seshadri et al., 2006 \\
\hline BWH65 & Non-vAh & Clinical & A. hydrophila & A. caviae & $\underline{\text { NZ_LESKO00000000.1 }}$ & Earl et al., 2015 \\
\hline E1 & Non-vAh & Clinical & A. hydrophila & A. hydrophila & $\underline{\text { SAMN01886638 }}$ & Grim et al., 2013 \\
\hline E2 & Non-vAh & Clinical & A. hydrophila & A. hydrophila & $\underline{\text { SAMN01886639 }}$ & Grim et al., 2013 \\
\hline GA97-22 & Non-vAh & Rainbow trout & A. hydrophila & Aeromonas spp. & $\underline{\text { SAMN01085627 }}$ & Hossain, 2012 \\
\hline $\mathrm{HZM}$ & Non-vAh & Soil & A. hydrophila & A. caviae & $\underline{\text { SAMN02596469 }}$ & Chua et al., 2015 \\
\hline WS & Non-vAh & Water sample & A. media & A. media & $\underline{\text { SAMN02472129 }}$ & Chai et al., 2012 \\
\hline $848^{\top}$ & Non-vAh & Wedge-shells & A. molluscorum & A. molluscorum & $\overline{\text { SAMN02471397 }}$ & Spataro et al., 2013 \\
\hline LMG24683 $^{\top}$ & Non-vAh & Unknown & A. taiwanensis & A. taiwanensis & SAMEA2752407 & Colston et al., 2014 \\
\hline MDS8 & Non-vAh & Dairy sludge & Aeromonas sp. & A. dhakensis & $\underline{\text { SAMN02472124 }}$ & Raychaudhuri et al., 2013 \\
\hline Ahy_Idx71 & vAh & Channel catfish & A. hydrophila & A. hydrophila & $\underline{\text { SAMN05292361 }}$ & This study \\
\hline AL09-71 & vAh & Channel catfish & A. hydrophila & A. hydrophila & NZ_CP007566.1 & Pridgeon et al., 2014 \\
\hline AL09-79 & vah & Channel catfish & A. hydrophila & A. hydrophila & $\underline{\text { NZ_LRRV00000000.1 }}$ & Hossain, 2012 \\
\hline ALG15-098 & vAh & Channel catfish & A. hydrophila & A. hydrophila & $\underline{\text { SAMN05223361 }}$ & This study \\
\hline IPRS15-28 & vAh & Channel catfish & A. hydrophila & A. hydrophila & $\underline{\text { SAMN05223362 }}$ & This study \\
\hline $\mathrm{J}-1$ & vAh & Crucian carp & A. hydrophila & A. hydrophila & NZ_CP006883.1 & Pang et al., 2015 \\
\hline JBN2301 & vAh & Crucian carp & A. hydrophila & A. hydrophila & $\underline{\text { NZ_CP013178.1 }}$ & Yang et al., 2016 \\
\hline ML09-119 & vAh & Channel catfish & A. hydrophila & A. hydrophila & NC_021290.1 & Liles et al., 2011 \\
\hline ML09-121 & vAh & Channel catfish & A. hydrophila & A. hydrophila & $\underline{N Z \text { LRRX000000000.1 }}$ & Hossain, 2012 \\
\hline ML09-122 & vAh & Channel catfish & A. hydrophila & A. hydrophila & NZ_LRRY00000000. & Hossain, 2012 \\
\hline ML10-51K & vAh & Channel catfish & A. hydrophila & A. hydrophila & $\underline{\text { SAMN05223363 }}$ & This study \\
\hline NJ-35 & vAh & Crucian carp & A. hydrophila & A. hydrophila & NZ_CP006870.1 & Pang et al., 2015 \\
\hline PB10-118 & vAh & Channel catfish & A. hydrophila & A. hydrophila & $\underline{\text { SAMN01085622 }}$ & Hossain, 2012 \\
\hline pc104A & vAh & Soil & A. hydrophila & A. hydrophila & NZ_CP007576.1 & Pridgeon et al., 2014 \\
\hline
\end{tabular}


TABLE 1 | Continued

\begin{tabular}{|c|c|c|c|c|c|c|}
\hline Strain & Phenotype & Isolation source & $\begin{array}{l}\text { GenBank species } \\
\text { assignation }\end{array}$ & $\begin{array}{l}\text { Species based on } \\
\text { phylogeny and ANI }\end{array}$ & Accession & Reference \\
\hline S04-690 & vAh & Channel catfish & A. hydrophila & A. hydrophila & $\underline{\text { SAMN02404466 }}$ & Hossain et al., 2014 \\
\hline S13-612 & vAh & Channel catfish & A. hydrophila & A. hydrophila & $\underline{\text { SAMN05292362 }}$ & This study \\
\hline S13-700 & vAh & Channel catfish & A. hydrophila & A. hydrophila & $\underline{\text { SAMN05292363 }}$ & This study \\
\hline S14-296 & vAh & Channel catfish & A. hydrophila & A. hydrophila & $\underline{\text { SAMN05292365 }}$ & This study \\
\hline S14-452 & vAh & Channel catfish & A. hydrophila & A. hydrophila & $\underline{\text { SAMN05256776 }}$ & This study \\
\hline S14-458 & vAh & Channel catfish & A. hydrophila & A. hydrophila & $\underline{\text { SAMN05223364 }}$ & This study \\
\hline S14-606 & vAh & Channel catfish & A. hydrophila & A. hydrophila & $\underline{\text { SAMN05292366 }}$ & This study \\
\hline S15-130 & vAh & Channel catfish & A. hydrophila & A. hydrophila & $\underline{\text { SAMN05223365 }}$ & This study \\
\hline S15-242 & vAh & Channel catfish & A. hydrophila & A. hydrophila & $\underline{\text { SAMN05223366 }}$ & This study \\
\hline$S 15-400$ & vAh & Channel catfish & A. hydrophila & A. hydrophila & $\underline{\text { SAMN05223367 }}$ & This study \\
\hline S15-591 & vAh & Channel catfish & A. hydrophila & A. hydrophila & $\underline{\text { SAMN05223368 }}$ & This study \\
\hline ZC1 & vAh & Grass carp & A. hydrophila & A. hydrophila & SAMN02404465 & Hossain et al., 2014 \\
\hline
\end{tabular}

Strains are indicated as virulent A. hydrophila (VAh) or other Aeromonas spp. based on their phylogenetic affiliation (Figure 1).

TABLE 2 | Oligonucleotide primers specific to members of the vAh pathotype (vAh-SerF and vAh-SerR), previously described qPCR vAh primers (2986F and 2986R), and primers used to screen for unique isolates used in this study (ML09-119F, ML09-119R, S14-452F, S14-452R, ZC1F, and ZC1R).

\begin{tabular}{lllc}
\hline $\begin{array}{l}\text { Primer } \\
\text { name }\end{array}$ & Direction & Sequence & $\begin{array}{c}\text { Amplicon } \\
\text { size (bp) }\end{array}$ \\
\hline vAh-SerF & Forward & 5'-AG'CATCACCAGCGTTGGCCC-3' $^{\prime}$ & 502 \\
vAh-SerR & Reverse & 5'-GCCGGGCTGAACTTCCGCAT-3' & \\
2986F & Forward & 5'-CTATTACTGCCCCCTCGTTC-3' & 167 \\
2986R & Reverse & 5'-ATTGAGCGGTATGCTGTCG-3' & \\
ML09-119F & Forward & 5'-GTTCCGTTCCATCTGTTCGTGA-3' & 246 \\
ML09-119R & Reverse & 5'-CAACCATCTTGGTCGCAATC-3' & \\
S14-452F & Forward & 5'-CAGAACGTGCTGCAGAGATTGA-3' & 350 \\
S14-452R & Reverse & 5'-TCCGAGAATCGATGACGAAGG-3' & \\
ZC1F & Forward & 5'-GCAATTCTGCGGTCACTTCTCG-3' & 400 \\
ZC1R & Reverse & 5'-AGCGTACCGTCTCGTCGATATG-3' & \\
\hline
\end{tabular}

final extension at $72^{\circ} \mathrm{C}$ for $5 \mathrm{~min}$. Amplicons were resolved on a $1 \%$ agarose gel and stained with ethidium bromide.

In order to have a PCR assay that differentiates vAh from non-vAh strains while encompassing both US and Asian vAh isolates, new vAh genotype-specific primers (listed as vAh-SerF and vAh-SerR in Table 2) were designed using comparative analysis of annotations produced by the Rapid Annotations using Subsystems Technology (RAST) v2.0 server. These primers target a serine protease gene sequence and are predicted to produce a 502 bp amplicon (data not shown). Touchdown PCR conditions and validation methods were identical to those described previously.

In addition to primers that help to identify vAh strains, A. hydrophila lineage-specific primers were developed that were specific to vAh lineages represented by strains ML09119 (NC_021290), S14-452 (SAMN05256776), and ZC1 (SAMN02404465) (Table 2). The ML09-119 lineage-specific primer set targets the tns $A$ endonuclease gene and produces a
246 bp amplicon, the S14-452 lineage-specific primer set targets the COG3339 genetic locus and produces a 350 bp amplicon, and the ZC1 lineage-specific primer set targets a hypothetical protein and produces a $400 \mathrm{bp}$ amplicon.

To determine the vAh genotype affiliation of disease isolates in $\mathrm{AL}$ and $\mathrm{MS}$, genomic DNA was isolated from each isolate (Gentra Puregene DNA isolation kit; Qiagen, Hilden, Germany) and used as a template in a $25-\mu$ l PCR that comprised of 13 $\mu l$ of Econotaq Plus Green 2x MasterMix (Lucigen, Madison, Wisconsin, USA), 20 picomoles of each oligonucleotide primer and $50 \mathrm{ng}$ of template gDNA. Samples were run on a C1000 Touch thermal cycler (BioRad, Hercules, California, USA) with an initial denaturation of $94^{\circ} \mathrm{C}$ for $3 \mathrm{~min}$ followed by 35 cycles of $94^{\circ} \mathrm{C}$ for $30 \mathrm{~s}, 58^{\circ} \mathrm{C}$ for $30 \mathrm{~s}$, and $72^{\circ} \mathrm{C}$ for $1 \mathrm{~min}$, with a final extension at $72^{\circ} \mathrm{C}$ for $5 \mathrm{~min}$ and amplicon verification was performed as previously described.

\section{Core Genome Analyses}

A core genome was created using both coding and noncoding sequences of 61 genomes labeled as A. hydrophila within GenBank, some of which were revealed to be misclassified. Specifically, any contigs less than $10 \mathrm{Kbp}$ in size were first filtered from draft genomes to increase computational efficiency and decrease false positives by limiting the mapping of smaller fragments to non-homologous and/or multiple regions. In general, smaller fragments contribute less to the production of core genomes as a function of being flanked by areas of heavily repetitive sequences. In the assembly stage, these regions stopped growing and remained short. While core genomes produced by this method are often smaller, the removal of smaller contigs did not compromise the ability to form a phylogeneticallyinformative core genome.

Filtered sequence data were then submitted as FASTA files to the multiple whole genome alignment tool Mugsy v1.2.3 (Angiuoli and Salzberg, 2011) under default parameters. The resulting alignment was subsequently processed with GBLOCK v0.91b (Castresana, 2000) in order to identify regions of 


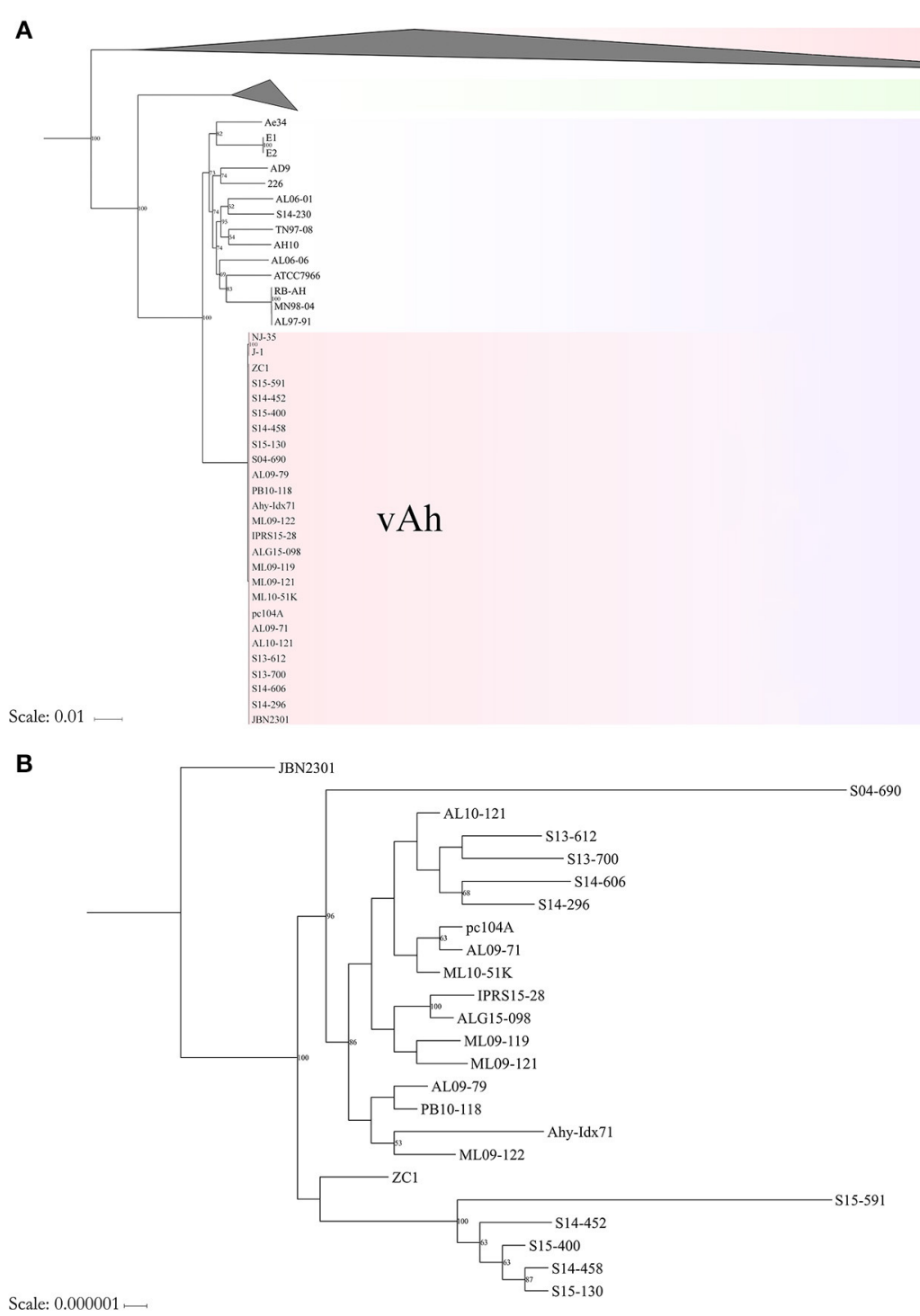

FIGURE 1 | Maximum likelihood (ML) phylogeny of (Panel A) Aeromonas spp. and (Panel B) vAh isolates based on the core genome of $3.78 \mathrm{Mb}$ conserved among these bacterial strains.

high conservation across all isolates. Parameters for retention by GBLOCK are dictated by the input alignment and were: A minimum of 31 and 51 sequences for conserved and flanked positions, respectively, a maximum of 8 contiguous, but non-conserved positions, a minimal block length of 10 , and one-half of the sequences were allowed to possess gapped positions within a block. From the final alignment, a maximum likelihood (ML) phylogeny for the 61 Aeromonas spp. isolates, including 54 isolates labeled in GenBank as A. hydrophila, was inferred using RAxML v8.2.8 (Stamatakis, 2014) under the General Time Reversible model of evolution with estimated proportions of invariable sites and rate variation among sites (i.e., $\mathrm{GTR}+\mathrm{I}+\mathrm{G}$ ) and 1000 bootstrap replicates to determine branch supports. Trees were visualized using Archaeopteryx v.beta 0.9901 .
Following generation of a consensus sequence, the National Microbial Pathogen Data Resource (NMPDR) Rapid Annotations using Subsystems Technology (RAST) v2.0 server was used in conjunction with the SEED v2.0 algorithm to annotate the core genome and generate metabolic models (Aziz et al., 2008; Overbeek et al., 2014). These predictive models were evaluated using both protein-protein Basic Local Alignment Search Tool (e.g., BLASTp and BLASTx) algorithms through GenBank as well as the Joint Genome Institute's Genomes OnLine Database (GOLD) v5.0.

\section{Calculating Average Nucleotide Identity}

To assess overall genetic similarity, the average nucleotide identity (ANI) comparison of 61 Aeromonas spp. genomes was evaluated using JSpecies (v1.2.1) and cross-validated with the 
Konstantinidis lab ANI calculator (Richter and Rosselló-Móra, 2009; Rodriguez and Konstantinidis, 2014). According to criteria used for the genus Aeromonas, ANI-values $>96 \%$ indicated strains belong to the same species (Colston et al., 2014; BeazHidalgo et al., 2015a).

\section{vAh Differential Gene Identification}

To evaluate differences in gene content among the 61 genomes, data from the PAthogen Resource Integration Center (PATRIC) protein family sorter tool, RAST/SEED gene annotations, the Pathogen Host Interaction database (http://www.phi-base.org), and the Virulence Factors of Pathogenic Bacteria databases (http://www.mgc.ac.cn/VFs) were combined with copy number data for previously identified virulence genes (Rasmussen-Ivey et al., 2016). Results were evaluated by comparing predicted virulence-associated genes with closely and disparately related isolates to identify genes that were differentially present. Notably, genes shared between $\mathrm{vAh}$ and non-vAh isolates or those unique to an individual strain were removed from downstream analyses. These data were subsequently coupled with screening of virulence factors and vAh-associated genes using searches of the NCBI GenBank database with MegaBLAST and BLASTn algorithms (Wheeler et al., 2003). Thresholds for absence were specific to the respective gene and were restricted to mutations altering the predicted functional domains of proteins in which the protein sequence in question returned a functionally divergent protein. Once validated, these gene clusters and virulence factors were transformed for statistical analyses using Orange Data Mining software v.3.3.5 and/or $\mathrm{R}$ Studio v.0.99.896. After pre-processing in R Studio using packages MuMIn v.1.15.6, randomForest v.4.6-12, and k-means v.0.1.1, heat maps of resultant data were generated using Orange Data Mining software. To analyze subclade differences, vAh and non-vAh strains with known virulence properties $(n=25)$ were evaluated using k-means at 20 clusters $(100 \%$ between sum of squares/total sum of squares). In addition to these analyses, the T346 Secretion System Hunter (version is not published) was used to identify T6SS-associated gene clusters using Glimmer v3.02, and HMMER3 v3.1b2 (Martínez-García et al., 2015).

\section{RESULTS}

\section{Identifying New vAh Isolates}

The S04-690 genome was previously found to be the genetic intermediate (raw genetic distance) between US catfish vAh isolates (represented by strain ML09-119) and the Asian carp vAh isolate ZC1 (Hossain et al., 2014). A total of $38 \mathrm{vAh}$ isolates recovered from MAS outbreaks in Mississippi from 2013 to 2015 and confirmed as vAh by phenotypic (myo-inositol usage) and/or genomic tests (qPCR) (Griffin et al., 2013; Hanson et al., 2014) were subjected to vAh lineage-specific PCR. Testing using lineage-specific primer sets based on representative isolates ML09-119, S14-452, and ZC1 showed that of the 38 MS isolates recovered from MAS outbreaks between 2013 and 2015, 20 isolates from a single farm were positive for the ML09-119 lineage-specific amplicon, while 18 isolates across five geographically discrete farms were positive for the S14-452 lineage-specific amplicon. No isolates produced amplicons with the ZC1 lineage-specific primer set (data not shown). Isolates that produced an amplicon with either the ML09-119 or S14452 lineage-specific primer sets did not produce amplicons when assayed with other lineage-specific primer sets (data not shown).

\section{Pathotype-Specific PCR for vAh Genotypes}

Results from touchdown PCR assays demonstrate that the original 2968 primer set (Griffin et al., 2013) reliably distinguishes US vAh from non-vAh isolates (data not shown). However, the Asian carp isolates J-1 and NJ-35 were unable to be experimentally evaluated and in silico analysis predicts that Asian isolates J-1 and NJ-35 would not produce a PCR amplicon using the 2968 primer set. To address this possible limitation, a new vAh genotype-specific primer set (listed as vAh-SerF and vAhSerR in Table 2) was designed to be inclusive of all Asian and US isolates, based on in silico analyses of vAh and non-vAh isolates. The vAh-SerF/R primer set was empirically confirmed to produce a $502 \mathrm{bp}$ amplicon in all available US vAh isolates while returning a negative result for non-vAh isolates (data not shown).

\section{Core Genome Analysis}

Alignment of the complete and filtered draft genomes of the 61 Aeromonas spp. genomes via Mugsy produced a matrix of $19,817,762$ positions. Following processing with GBLOCK, the core genome of these 61 strains contained 32,401 blocks and a consensus of 3,776,490 bp. This included 120,049 variable ( $>2$ different nucleotides) and 79,507 phylogenetically informative sites (nucleotides that contributed to sorting phylogenetic groups), with percent $\mathrm{G}+\mathrm{C}$ composition of $62.6 \%$. Notably, these conserved regions collectively have a higher percent $\mathrm{G}+\mathrm{C}$ content than the 61 -isolate average of $61.1 \%$ (with a range of $60 \%$ for the genome of the type strain of A. molluscorum $848^{\mathrm{T}}$ to $63.2 \%$ for the one of $A$. taiwanensis LMG24683 ${ }^{\mathrm{T}}$; $p$-value $=$ 0.003). Across the core analysis, within complete sites, the average transition to transversion ratio was 1.437 for all sequence pairs, with a minimum of 0 transitions and 1 transversion (A. hydrophila S04-690 and S13-700) and a maximum of 13 transitions and 2 transversions (A. hydrophila 173 and 14).

\section{Core Genome Phylogeny}

Conserved sequences from the core genome analysis were used to infer phylogenetic relationships among the 61 Aeromonas spp. isolates (Figure 1A). The core genome phylogeny indicates with $100 \%$ bootstrap support that vAh strains form a monophyletic group that is fundamentally distinct from other A. hydrophila (Figure 1A). In addition to supporting the monophyly of these ST251 isolates, this phylogenetic analysis also revealed support for distinct sub-clades among vAh isolates, with 2660 single nucleotide polymorphisms identified between the vAh subclades. For example, A. hydrophila ZC1, isolated from a grass carp in China (Figure 1B), is closely affiliated with vAh strains isolated from catfish in MS (S14-452, S14-458, S15-130, S15-400, and S15-591). A key genotype that differentiates the vAh sub-clades is the presence and genetic organization of T6SS components (Figure 2). Each of the ZC1-affiliated strains, as well as the other strains isolated from carp in China (i.e., J-1 and NJ-35), were 


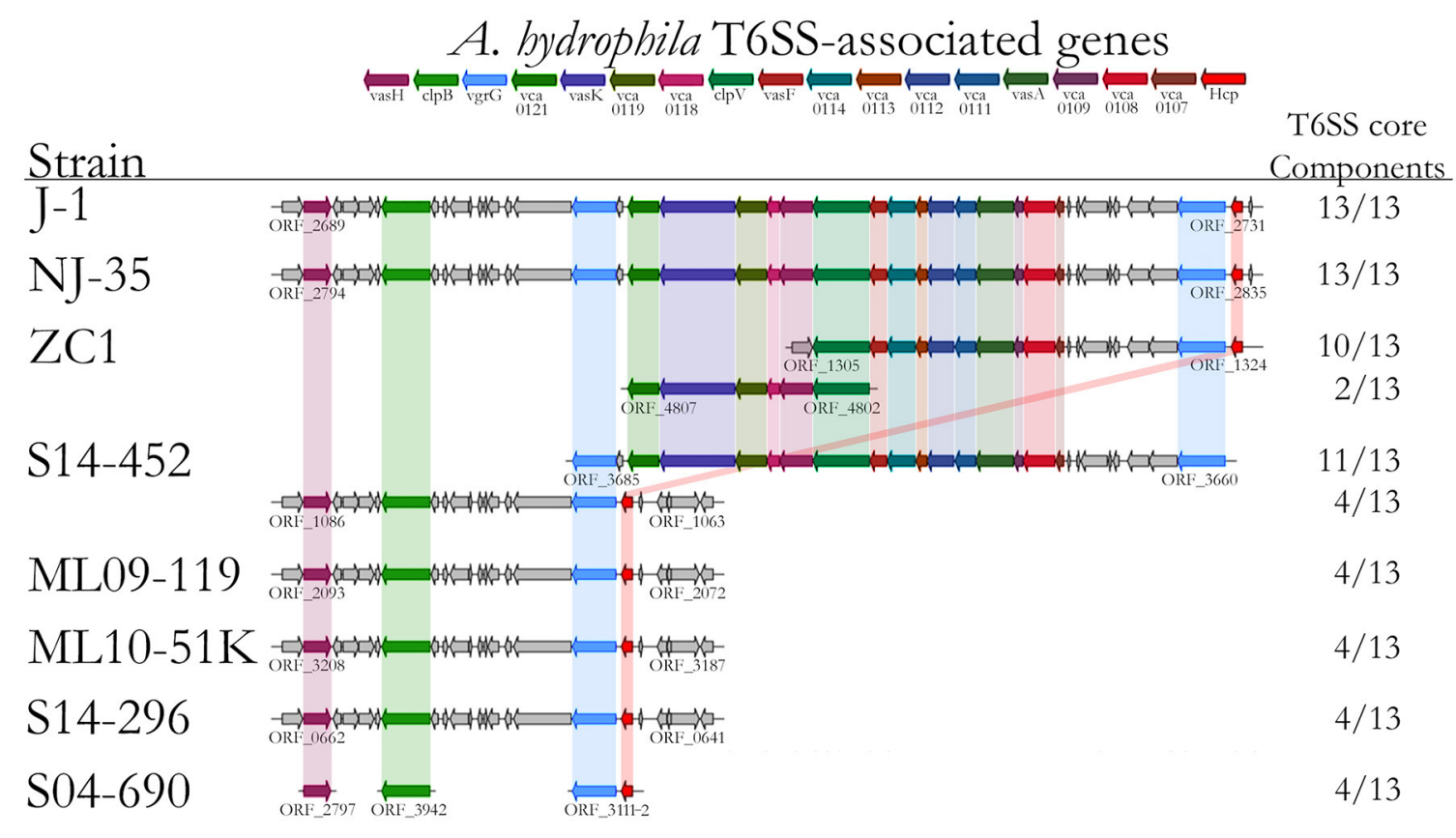

Scale: ${ }_{5 \mathrm{k} b}$

FIGURE 2 | Type VI secretion system gene prediction using the T346 Secretion System Hunter, with results including strains included in the immersion catfish challenge (ML09-119, MNL10-51K, S04-690, S14-296, S14-452, and ZC1) and representatives from Chinese strains (J-1, NJ-35, and ZC1).

found to contain at least $80 \%$ of the core proteins necessary for a complete T6SS, with ZC1 and S14-452 having two separate T6SSassociated gene clusters, whereas J-1 and NJ-35 each have a single complete T6SS cluster (Figure 2). In contrast, vAh isolates from catfish in AL as well as other MS isolates (i.e., S13-612, S13-700, S14-606, and S14-296) formed a distinct subclade that lack the majority of the core T6SS genes (Figures 1B, 2). Notably, while AL and MS vAh strains lacked the majority of T6SS components, they consistently retain other genes that are involved in T6SS in other bacteria such as a valine-glycine repeat protein $\mathrm{G}$ (VgrG), a T4 bacteriophage tail-like hole forming protein (Leiman et al., 2009); hemolysin coregulated protein (Hcp), a repetitive tubular protein that is similar to the phage major tail protein GpV (Pell et al., 2009); the chaperone protein $\mathrm{ClpB}$, a chaperone and ATPase that interacts with Hcp to translocate effectors (Shrivastava and Mande, 2008); and VasH, a putative transcriptional regulator (Kitaoka et al., 2011) (Figure 2).

\section{Average Nucleotide Identity}

The average nucleotide identity values of the 61 Aeromonas spp. genomes was determined (Figure 3). High ANI-values (>99\%) were found for all vAh-vAh pairwise comparisons, supporting the core genome phylogeny (Figures 1B, 3). In contrast, all vAh comparisons with non-vAh isolates possessed ANI-values less than $97 \%$. In accordance with previous results, 14 strains appear to have a discrepancy between the species classification listed in GenBank and the species affiliation indicated by ANI-values (Table 1).

\section{Virulence of vAh Strains in Channel Catfish Using an Immersion Challenge Model}

Challenge of channel catfish with vAh isolates from AL and MS resulted in $\geq 60 \%$ mortality (Figure 4 ). Within these US vAh isolates tested, there were no observed differences in virulence among ML09-119, ML10-51K, S04-690, S14-296, and S14-452, based on Duncan's multiple range test ( $p$-value $>0.05$ ). The carp isolate $\mathrm{ZC1}$ was less virulent than $\mathrm{AL}$ and $\mathrm{MS}$ isolates with only 26.7\% mortality observed (Figure 4). Most mortality ( 96\%) occurred within $48 \mathrm{~h}$ post challenge for all isolates, including ZC1. All dead fish (100\%) sampled for confirmation were positive for the presence of vAh in liver tissue. Control fish yielded no mortality from the mock challenge.

\section{Pathology of Epidemic A. hydrophila Infections}

Cutaneous lesions observed in fish infected with vAh by immersion challenge included extensive hyperemia over the pale ventrum of the fish, in tissues surrounding and within the mouth and at fin bases (Figure 5A). There was also extensive hyperemia around the eyes and exophthalmos in some fish. Cutting into the muscle of the lateral body wall revealed multifocal to coalescing foci of congestion/hemorrhage. Gill lesions were variable; gills were pale in some fish and reddened in others. Internally, there was widespread hyperemia of abdominal organs as well as petechial and ecchymotic hemorrhages scattered over mesenteric tissues. The spleen was moderately to severely swollen and 


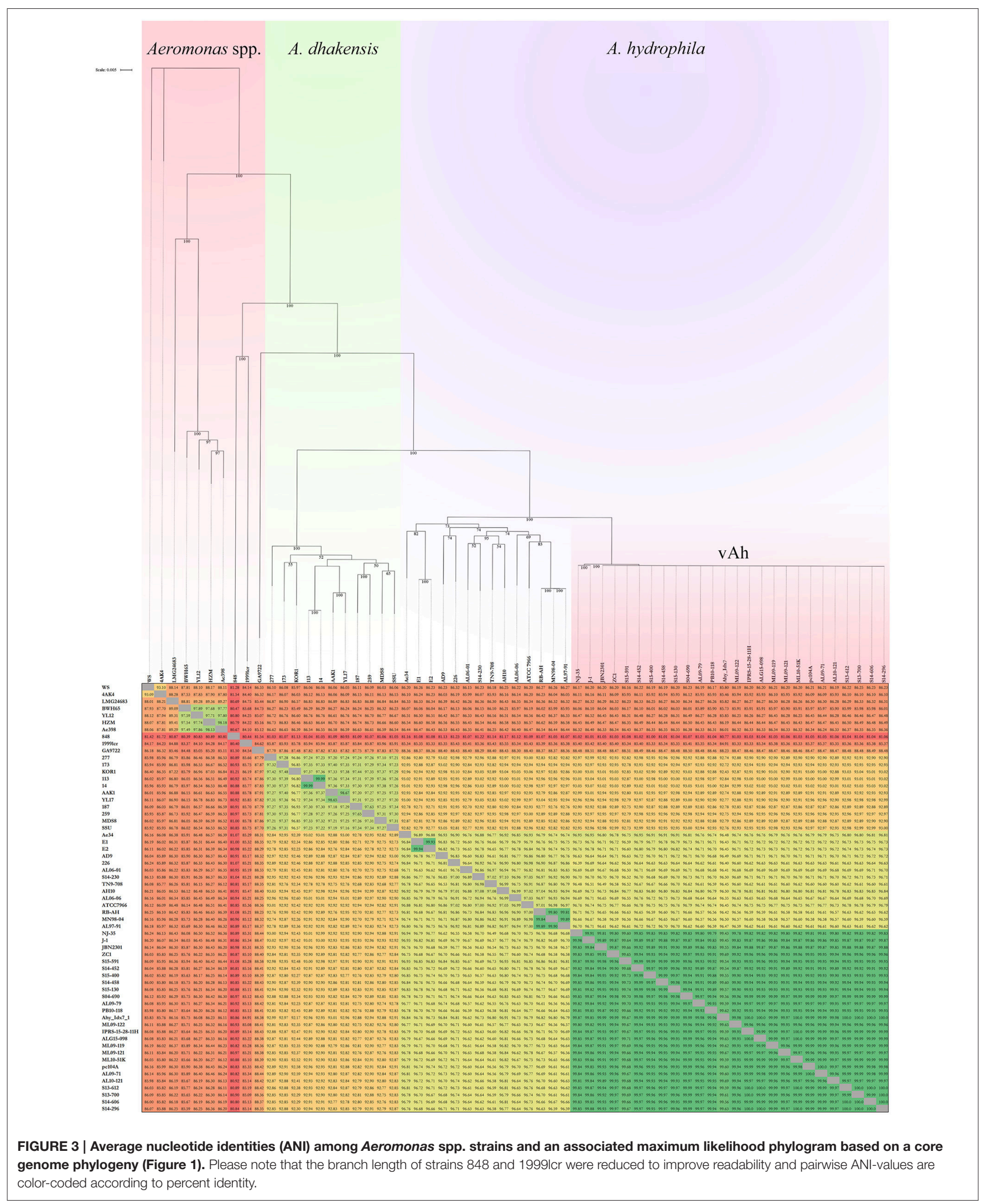




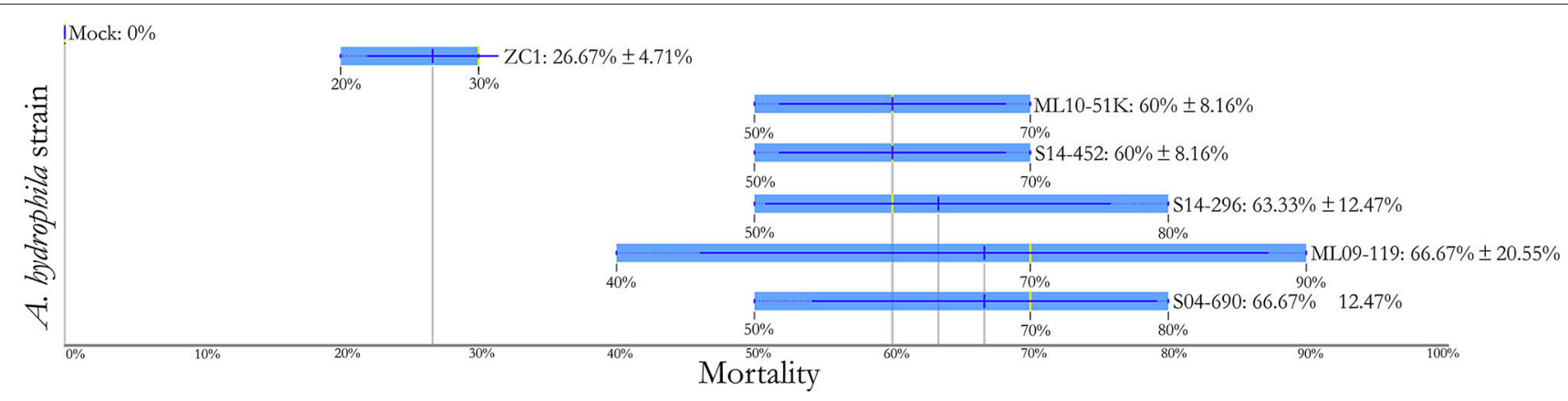

FIGURE 4 | Comparative assessment of the relative virulence of vAh isolates in channel catfish using $1 \mathrm{~h}$ immersion exposure with fin clip (ANOVA = 7.628, $p$-value $=0.001$ ).
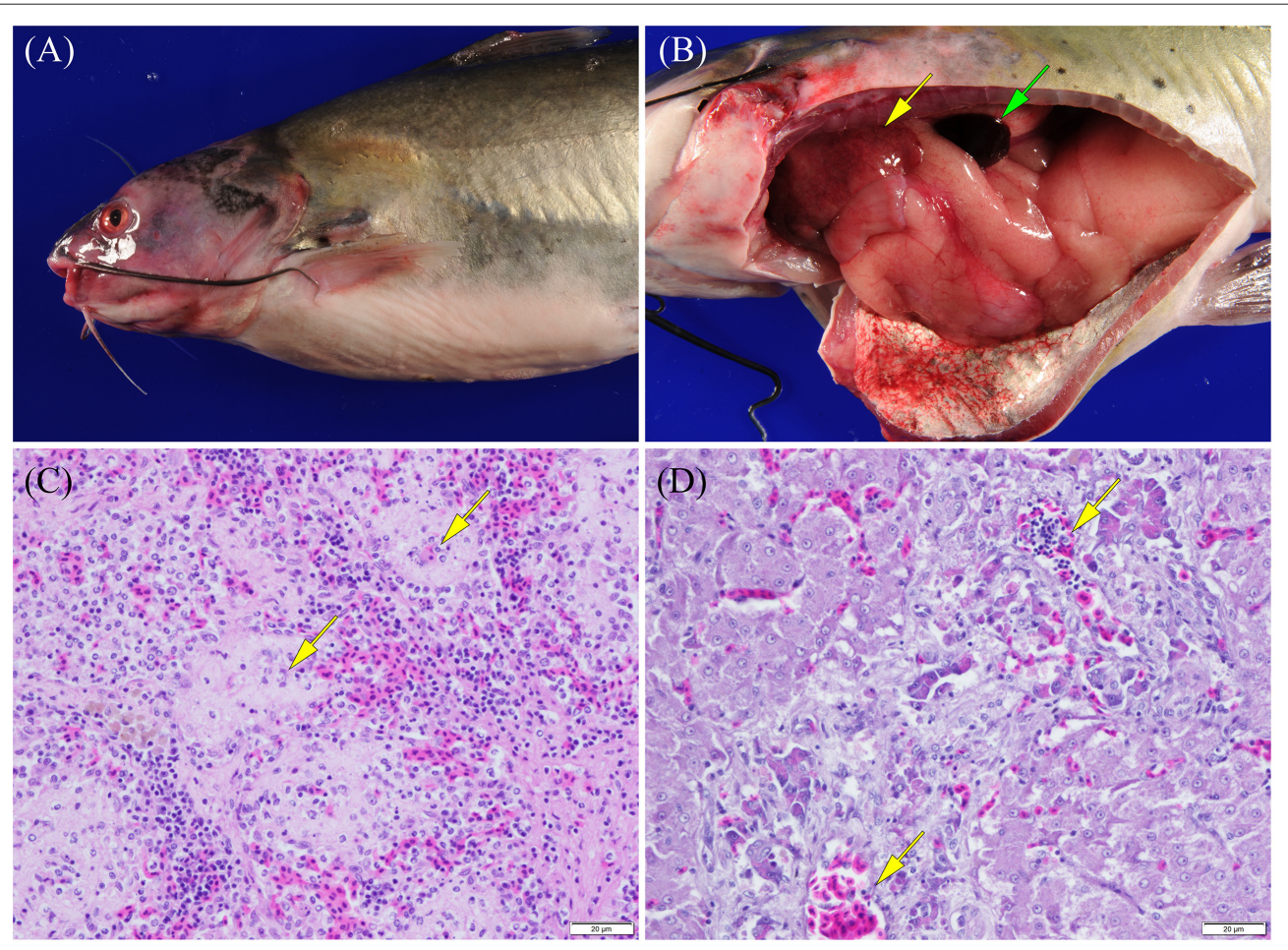

FIGURE 5 | Photographs of channel catfish infected by vAh showing (A) external surfaces that are exhibiting congestion/hemorrhage around the head/pectoral fin and within the eye and (B) the celomic cavity that has internal organs moderately congested and enlarged, a

congested/hemorrhagic spleen (green arrow), and multifocal pale foci corresponding to areas of necrosis (yellow arrow) scattered over the liver (photographs courtesy of Dr. Wes Baumgartner, Mississippi State University) as well as photomicrographs of a channel catfish infected by vAh strain ML09-119 showing (C) a section of spleen with splenic ellipsoids (arrows) that are edematous and ellipsoidal arteries that are lined by degenerating as well as necrotic endothelial cells and (D) a section of liver with edema and necrosis of pancreatic acinar tissue surrounding branches of the hepatic portal vein (arrows).

dark red (Figure 5B). Head and trunk kidneys were moderately edematous and red and friable when harvested. The intestinal tract was mildly to moderately dilated and red (Figure 5B). The liver was mildly to moderately swollen with slightly rounded edges (Figure 5B). Glisson's capsule was peppered with variable numbers of petechial and ecchymotic hemorrhages. The atrial chamber of the heart was dilated and filled with blood.

Histopathologic lesions observed were strongly suggestive of a septic disease and included edema and necrosis in many internal organs. Splenic lesions included ellipsoidal necrosis and congestion/hemorrhage in the splenic red pulp (Figure 5C). In scattered necrotic ellipsoids variable numbers of short, rodshaped bacteria were observed. In the liver there was often necrosis of the acinar pancreatic tissue surrounding hepatic vessels (Figure 5D). Scattered acinar cells were rounded and necrotic and extracellular zymogen granules could be observed in the necrotic exudate. Small numbers of inflammatory cells including macrophages, lymphocytes, and neutrophils were 
observable in scattered areas of pancreatic acinar necrosis. Multifocally within the hepatic parenchyma were variable sized foci of hepatocellular necrosis characterized by the presence of small aggregates of degenerating and necrotic hepatocytes. Hematopoietic cells in the renal interstitum and renal epithelial cells lining scattered renal tubules were undergoing degeneration and necrosis. In sections of head kidney, the tissue was edematous and congested/hemorrhagic. There was scattered degeneration and necrosis of red and white cell precursors. Intestinal lesions were minimal in fish infected in this fin clip model and were limited to mild congestion and hemorrhage of the vessels in the lamina propria and vessels of the muscularis and serosa. In some sections of heart there was mild necrosis of myofibers in the myocardium. Vessel of the brain were often moderately dilated and congested but the neuropil of the cerebrum, cerebellum, and brain stem was normal. The epithelium of the gills was normal but branchial capillaries were sometimes dilated and congested with erythrocytes.

\section{Differentiating Pathotypes}

In order to robustly define the vAh pathotype-specific loci in this study, previous results on established $A$. hydrophila virulence factors as well as genes unique to vAh strains were used in combination with a clustering approach and a random forest decision tree to identify gene products that may contribute to functional differences in virulence. This approach confirmed previously described gene clusters for L-fucose and O-antigen biosynthesis as well as myo-inositol catabolism. Additionally, we identified predicted virulence factors conserved within all vAh strains (Table 3) which includes virulence factors well-known to be important in A. hydrophila pathogenesis (RasmussenIvey et al., 2016). Among predicted virulence factors conserved among vAh strains, there were many genes uniquely associated with vAh strains that were not present in other sequenced A. hydrophila strains, including a L-serine dehydratase, a Nacetylmannosamine kinase, a $\mathrm{N}$-acetylneuraminate lyase, and a gene product predicted to be required for queuosine biosynthesis (Table 4). In a more comprehensive approach, all RAST/SEED predicted genes from the 41 confirmed A. hydrophila genomes (26 vAh and 15 non-vAh) included in this study were evaluated on the basis of linkage with the vAh pathotype using exhaustive iterations of random forest modeling, which resulted in 26 genes uniquely associated with vAh by either presence/absence or by differential copy number when compared to non-vAh (Figure 6).

\section{DISCUSSION}

The core genome analysis of epidemic A. hydrophila strains obtained from diseased fish from several US states and Chinese provinces (identified within the literature as ST251/vAh), supports the genetic and functional unification of these hypervirulent bacteria within a monophyletic clade. These data are in agreement with previous reports based on single or multiple genetic loci from smaller numbers of vAh strains (Hossain et al., 2014; Pang et al., 2015). Interestingly, this study found evidence for genomic heterogeneity among the sampled vAh strains that may reflect geographic origin and/or host switching. For example, the isolates obtained from MS in 2013-2015 are affiliated with two different ST251/vAh clades, specifically the Asian carp-affiliated clade and the US catfishaffiliated clade. In contrast, the vAh isolates from diseased catfish in AL from 2009 to 2015 reflect a single, clonal clade. These data suggest that vAh has greater diversity within MS aquaculture ponds compared to those of AL. As previously hypothesized, this pattern would fit with a dissemination model in which carp or other fish or fish products from Asian source(s) were first introduced to the Mississippi delta region, after which a particularly more virulent vAh lineage spread among farmed catfish resulting in the initial epidemic outbreaks within AL.

Genomic comparisons indicate all members of the vAh pathotype strains share unique genetic loci that may be a result of their close genetic affiliation and may also contribute to their pathogenesis. The previously identified vAh-specific gene clusters of L-fucose and myo-inositol catabolism were also confirmed in this study. The use of myo-inositol as a sole carbon source is a rarely observed phenotype among Aeromonas species and, to our knowledge, has only been reported in vAh strains and strains of A. finlandiensis (Beaz-Hidalgo et al., 2015b). This study identified additional genetic loci that are present in all sequenced vAh strains and may have a contribution to virulence, such as a L-serine dehydratase, a $\mathrm{N}$-acetylmannosamine kinase, a $\mathrm{N}$-acetylneuraminate lyase, a sialic acid transporter, a transcriptional regulator of pyridoxine metabolism, an archaeosine tRNA-ribosyltransferase, a gene product required for queuosine biosynthesis (QueD), an acriflavine resistance protein $\mathrm{A}$, and an IS5 transposase and transactivator. While the contribution of each of these gene products to $\mathrm{vAh}$ virulence has yet to be determined, studies in other bacterial pathogens suggest potential mechanisms that could enhance vAh virulence. For example, in Campylobacter jejuni a L-serine dehydratase is essential for gut colonization (Velayudhan et al., 2004). With regard to sialic acid, Vibrio cholera has been shown to use this system to evade the innate immune response (Severi et al., 2007), and promote the binding of cholera toxin to the host intestinal epithelium (Rohmer et al., 2011). In addition, increased acriflavine resistance may provide a selective advantage to $\mathrm{vAh}$ considering that acriflavine is a commonly used antiseptic in aquaculture (Martin, 1968).

All annotated genes were subsequently evaluated for linkage with the vAh genotypes, a method that removes the bias inherent in assuming that only known virulence factors contribute to vAh pathogenesis. This analysis revealed that 26 genes are synonymous with the vAh pathotype either by presence/absence or by copy number. Supporting the robust nature of this approach, previously described genes that are vAh-associated were also identified, including genes associated with the myo-inositol catabolic pathway. Of note, within this pathway a methylmalonate-semialdehyde dehydrogenase that is required for myo-inositol catabolism was identified in both vAh and non-vAh isolates; however, this enzyme is required for both myo-inositol and valine metabolism and there is no indication that non-vAh strains have the genetic capacity for myo-inositol catabolism. In addition to these results, this method also identified known Aeromonas spp. virulence factors, such as a 
TABLE 3 | Predicted virulence factors that are conserved within vAh strains (not unique to), based on a comparison of significant BLASTn hits between vAh isolates against the VFDB (virulence factors with additional results are marked with an asterisk and are available in supplementary data).

\begin{tabular}{|c|c|c|c|c|}
\hline Putative virulence factor & Gene & Uniprot ID & GI & Reference bacterium \\
\hline 3-oxoacyl-acyl carrier protein synthase II & $f a b B$ & $\mathrm{AOAOH} 2 \mathrm{~V} 610$ & 77416726 & Escherichia coli O6:H1 \\
\hline Acriflavine resistance protein AcrB & acrB & P31224 & 25009252 & Escherichia coli K12 \\
\hline Aerolysin/hemolysin/cytolytic enterotoxin & ahh & Q06303 & 89276735 & Aeromonas hydrophila $\mathrm{AH}-1$ \\
\hline Asparaginyl-tRNA synthetase & asns & Q56112 & 16502162 & Salmonella enterica serovar Typhi CT18 \\
\hline Cephalosporinase; class C beta lactamase & ampC & Q8KU09 & 21311545 & Aeromonas caviae CIP 74.32 \\
\hline Enterochelin esterase & fes & $\mathrm{AOAOH} 2 \mathrm{~V} 760$ & 26106962 & Escherichia coli O6:H1 \\
\hline Ethanolamine utilization protein EutN & eutN & B7LTU3 & 984388511 & Escherichia fergusonii ATCC 35469 \\
\hline Flagellar motor switch protein FliN & fliN & AOAOH3QVI1 & 674744044 & Pseudomonas aeruginosa Stone 130 \\
\hline General secretion pathway protein PulF & pulF & P15745 & 149305 & Klebsiella pneuoniae oxytoca UNF5023 \\
\hline Protein translocase subunit SecA & $\sec A$ & Q8YJG2 & 672757090 & Brucella melitensis biotype 1 \\
\hline Rod shape-determining protein MreB & mreB & P0A9X4 & 557273544 & Escherichia coli K12 \\
\hline Sodium/proline symporter proline permease & putP & P07117 & 131658 & Escherichia coli K12 \\
\hline Transcriptional activator NtrC & ntrC & 086057 & 5731350 & Herbaspirillum seropedicae DCP286A \\
\hline Twitching motility protein PilU & pill & G3XCX3 & 15595593 & Pseudomonas aeruginosa PAO1 \\
\hline
\end{tabular}

TABLE 4 | Virulence factors that are unique to vAh strains, based on a comparison of significant BLASTn hits between A. hydrophila isolates against the RAST/SEED database.

\begin{tabular}{|c|c|c|}
\hline Subsystem & Role & GI \\
\hline Glycine/serine Utilization & L-serine dehydratase & 958619257 \\
\hline Inositol catabolism & 5-deoxy-glucuronate isomerase & 958618826 \\
\hline Inositol catabolism & 5-keto-2-deoxygluconokinase & 827371814 \\
\hline Inositol catabolism & Epi-inositol hydrolase & 612156152 \\
\hline Inositol catabolism & Inositol transport system ATP-binding protein & 958621246 \\
\hline Inositol catabolism & Inositol transport system permease protein & 958620586 \\
\hline Inositol catabolism & Inositol transport system sugar-binding protein & 657060685 \\
\hline Inositol catabolism & Inosose dehydratase & 507222178 \\
\hline Inositol catabolism & Myo-inositol 2-dehydrogenase 1 & 656991783 \\
\hline Inositol catabolism & Myo-inositol 2-dehydrogenase 2 & 827371809 \\
\hline Inositol catabolism & Transcriptional regulator of the myo-inositol catabolic operon & 958618669 \\
\hline Sialic Acid Metabolism & $\mathrm{N}$-acetylmannosamine kinase & 827373367 \\
\hline Sialic Acid Metabolism & $\mathrm{N}$-acetylneuraminate lyase & 1043232173 \\
\hline Sialic Acid Metabolism & Predicted sialic acid transporter & 446588390 \\
\hline Sialic Acid Metabolism & Sugar isomerase involved in processing of sialic acid & 958620857 \\
\hline Pyridoxin Biosynthesis & Predicted transcriptional regulator of pyridoxine metabolism & 16078013 \\
\hline Phage DNA synthesis & DNA adenine methyltransferase, phage-associated & 67483065 \\
\hline Phage capsid proteins & Phage capsid scaffolding protein & 516389014 \\
\hline Phage capsid proteins & Phage major capsid protein & 507220251 \\
\hline Phage lysis modules & Phage lysin, 1,4-beta- $\mathrm{N}$-acetylmuramidase & 511291760 \\
\hline Phage packaging machinery & Phage portal protein & 958618794 \\
\hline Phage packaging machinery & Phage terminase small subunit & 759443491 \\
\hline Phage packaging machinery & Phage terminase, large subunit & 958620694 \\
\hline Queuosine-Archaeosine Biosynthesis & Queuosine biosynthesis QueD, PTPS-I & 1043232409 \\
\hline Queuosine-Archaeosine Biosynthesis & archaeosine tRNA-ribosyltransferase type 5 & 507221161 \\
\hline
\end{tabular}

DNA adenine methylase that has been identified as a regulator of virulence and required for viability in A. dhakensis SSU (Erova et al., 2006). From this approach other putative vAh virulence factors were identified, such as a hypothetical protein with a LuxR-like domain. LuxR has been previously shown to be a regulator of virulence factors and quorum sensing within Aeromonas spp. (Kirke et al., 2004). Lastly, other genetic loci were identified as being associated with vAh strains, such as the type II/IV system secretin RcpA/CpaC that is putatively involved in flp pilus assembly (Clock et al., 2008), but to our knowledge the 


\section{Copy number}

$\begin{array}{llll}1 & 2 & 3 & 4\end{array}$

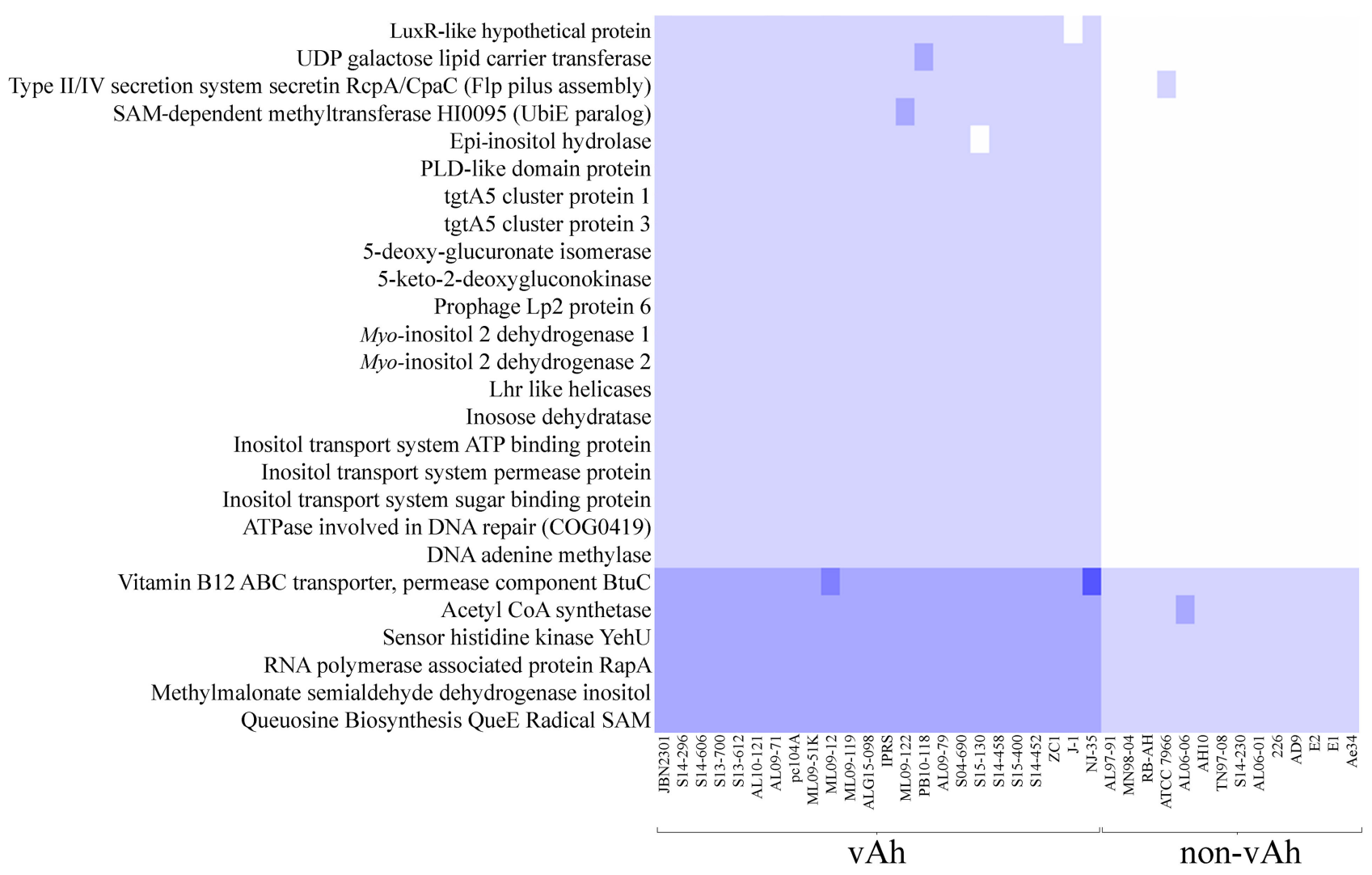

FIGURE 6 | Comparative whole genome predicted gene-based analysis of all confirmed vAh $(n=26)$ and non-vAh isolates $(n=15)$.

contribution of this secretin to A. hydrophila pathogenesis has yet to be experimentally determined.

The histopathologic examination of tissues from vAhinfected farmed fish show a wide range of severity related to internal lesions, with some fish exhibiting minimal lesions and others having widespread sepsis with necrosis of spleen, liver, renal tissue, intestine, and brain tissues with subsequent high rates of mortality occurring throughout affected farms (W. Hemstreet, personal communication). For fish challenged with vAh strains in aquaria via intraperitoneal injection, rapid onset of mortality without these disease sequelae was observed (Hossain et al., 2014). In this study, fish were challenged with an immersion model and exhibited significant clinical signs including cutaneous and ocular hemorrhaging, splenic and renal congestion, and hemorrhage with mild to moderate necrosis of internal organs. Despite the high genetic similarity of the strains (ANIs > 99\%), strain ZC1 had reduced virulence $(\sim 27 \%$ mortality) when compared with ML09-119; ML10-51K; S04690; S14-296; and S14-452 which caused $\geq 60 \%$ mortality in channel catfish. The reduced virulence of strain $\mathrm{ZC1}$, relative to strain ML09-119, in carp and in catfish was previously observed when fish were challenged intraperitoneally (Hossain et al., 2014). Interestingly, comparatively few reports of mortalities due to
MAS have come from the state of MS, which may reflect a number of geographic differences, the heterogeneity of vAh strains present within MS aquaculture ponds, differences in management and production practices, and/or environmental conditions.

To identify genetic elements that may explain the disparity in virulence between the Asian carp isolate ZC1 and the US catfish isolates, genomic comparisons were conducted between $\mathrm{ZC1}$ and the vAh strains included in the immersion challenge. These analyses revealed that all other vAh isolates tested in the disease challenge, but not strain ZC1, encode two putative transcription regulators: (1) a mobile element protein in the helix-turn-helix (HTH) superfamily and (2) a phage antirepressor protein in the antA superfamily present in Escherichia coli ECOR-9 (Sandt et al., 2002). The presence of these transcription factors within the highly virulent vAh strains could result in increased virulence factor expression in these strains. Furthermore, vAh isolates from Asian carp or the ZC1-affiliated clade from MS (including S14-452) were found to have a potentially functional T6SS, but the clade comprised solely of AL and MS catfish isolates (e.g., ML09-119, ML10-51K, S04-690, and S14-296) were found to consistently lack 9 of 13 core T6SS genes related to secretion of virulence factors such as Hcp. The observation that the isolates 
in the AL/MS vAh subclade lack a complete and/or functional T6SS would lead one to hypothesize that virulence is attenuated in these vAh strains; however, our experimental results indicate that these AL/MS disease isolates are highly virulent compared to strain ZC1. Previous disease challenges with strain ZC1 using intraperitoneal injection support the conclusion that this Asian carp strain $\mathrm{ZC} 1$ has reduced virulence relative to the AL/MS vAh clade in both catfish and carp (Hossain et al., 2014), indicating that the attenuated virulence observed in strain $\mathrm{ZC1}$ is not solely attributable to host-specific differences. The vAh strains in the AL/MS subclade encode only a subset of T6SS components that include VgrG, Hcp, ClpB, and VasH. The absence of the other T6SS core genes, including vca0107-0109, vca0111-0114, $v c a 0118, v c a 0119, v c a 0121, v a s K, c l p V, v a s F$, and vasA, largely accounts for the $\sim 16 \mathrm{Kbp}$ net difference in the genome sizes between strains ZC1 and ML09-119. Based on studies of T6SS in Aeromonas dhakensis SSU and in Salmonella enterica serovar Typhi (Suarez et al., 2008; Wang et al., 2011), it is hypothesized that US isolates lacking several T6SS proteins would exhibit better evasion of the host's immune response. Future research should aim to elucidate the role of these T6SS components, and the effect of a reduced or rearranged T6SS, on vAh virulence.

As new vAh isolates emerge and our collective knowledge of vAh genomic diversity grows, future research should investigate the spread of these pathogens as well as improve the design of more effective biosecurity strategies for the aquaculture industry. Toward this effort, we report a primer set that differentiates vAh from non-vAh strains and is inclusive of the known diversity of vAh strains in the US and Asia. The combination of tools available to differentiate vAh strains from non-vAh strains, including $\mathrm{qPCR}$ and the myo-inositol growth assay, are vital tools that can be used to map the global distribution of vAh in carp, catfish, and other warm-water fish species (e.g., tilapia). Disease control strategies should take into account the variability observed in this study among vAh strains and evaluate the efficacy of vaccination or other control measures against a panel of strains that represent the known diversity of this highly virulent $A$. hydrophila pathotype.

In conclusion, hypervirulent $A$. hydrophila within ST251 have emerged as pathogens of farmed warmwater fishes that are classified within the vAh pathotype based on strong phylogenetic evidence that includes a core genome phylogeny and ANIvalues $>99 \%$; metabolic activities that are unique within

\section{REFERENCES}

Angiuoli, S. V., and Salzberg, S. L. (2011). Mugsy: fast multiple alignment of closely related whole genomes. Bioinformatics 27, 334-342. doi: 10.1093/bioinformatics/btq665

Aziz, R. K., Bartels, D., Best, A. A., DeJongh, M., Disz, T., Edwards, R. A., et al. (2008). The RAST server: rapid annotations using subsystems technology. BMC Genomics 9:75. doi: 10.1186/1471-2164-9-75

Beatson, S. A., das Graças de Luna, M., Bachmann, N. L., Alikhan, N. F., Hanks, K. R., Sullivan, M. J., et al. (2011). Genome sequence of the emerging pathogen Aeromonas caviae. J. Bacteriol. 193, 1286-1287. doi: 10.1128/JB.01337-10

Beaz-Hidalgo, R., Hossain, M. J., Liles, M. R., and Figueras, M. J. (2015a). Strategies to avoid wrongly labelled genomes using as example the detected wrong this species, such as myo-inositol and sialic acid metabolism; a suite of conserved Aeromonas spp. virulence factors; 26 conserved genetic loci putatively linked with virulence; and the ability to induce motile Aeromonas septicemia, which is characteristically followed by rapid mortality in multiple species of farmed fish. Collectively, these traits distinguish vAh from non-epidemic A. hydrophila and define the vAh pathotype.

\section{AUTHOR CONTRIBUTIONS}

CR generated and analyzed genome sequence data; CR and SO analyzed gene copy number for putative virulence factors; JT and $\mathrm{WH}$ isolated vAh disease isolates from $\mathrm{AL}$ and collected data on fish disease; CS, DZ, and DX conducted vAh disease trials; $\mathrm{CR}$ and $\mathrm{MH}$ developed vAh-specific primer sets; MG collected disease isolates from Manuscript and typed them using different primer sets and bioassays, YL collected disease isolates from Chinese carp and analyzed data, MF contributed to the phylogenetic analysis and inositol utilization of Aeromonas isolates, SS conducted the core genome phylogenetic analysis, JN conducted the histology analysis and contributed to the writing and editing of the manuscript; and ML contributed to the genome sequence analysis, organizing the different research activities and to the writing and editing of the manuscript.

\section{FUNDING}

This work was supported by a grant from the United States Department of Agriculture's Agriculture and Food Research Initiative (Projects \#2013-67015-21313 and \#MIS-371530) and the USDA-ARS (CRIS Project No. 6010-32000-026-00D and the Catfish Health Initiative). MF thanks the support by the projects: Aquavalens from the European Union Seventh Framework Program (FP7/2007-2013) under Grant agreement No.: 311846, and by projects from Spanish Ministry of Science and Innovation: AGL2011-30461-C02-02 and JPIW2013-095-CO3.

\section{SUPPLEMENTARY MATERIAL}

The Supplementary Material for this article can be found online at: http://journal.frontiersin.org/article/10.3389/fmicb. 2016.01615 taxonomic affiliation for Aeromonas genomes in the genbank database. PLoS ONE 10:e0115813. doi: 10.1371/journal.pone.0115813

Beaz-Hidalgo, R., Latif-Eugenín, F., Hossain, M. J., Berg, K., Niemi, R. M., Rapala, J., et al. (2015b). Aeromonas aquatica sp nov., Aeromonas finlandiensis sp nov and Aeromonas lacus sp nov isolated from Finnish waters associated with cyanobacterial blooms. Syst. Appl. Microbiol. 38, 161-168. doi: 10.1016/j.syapm.2015.02.005

Beaz-Hidalgo, R., Martínez-Murcia, A., and Figueras, M. J. (2013). Reclassification of Aeromonas hydrophila subsp. dhakensis Huys et al. 2002 and Aeromonas aquariorum Martinez-Murcia et al. 2008 as Aeromonas dhakensis sp. nov. comb nov. and emendation of the species Aeromonas hydrophila. Syst. Appl. Microbiol. 36, 171-176. doi: 10.1016/j.syapm.2012. 12.007 
Camus, A. C., Durborow, R. M., Hemstreet, W. G., Thune, R. L., and Hawke, J. P. (1998). Aeromonas Bacterial Infections: Motile Aeromonad Septicemia (No. 478). Southern Regional Aquaculture Center (Southern Regional Aquaculture Center collectively covers Alabama, Arkansas, Florida, Georgia, Kentucky, Louisiana, Mississippi, North Carolina, Oklahoma, Puerto Rico, South Carolina, Tennessee, Texas, U.S. Virgin Islands, and Virginia).

Castresana, J. (2000). Selection of conserved blocks from multiple alignments for their use in phylogenetic analysis. Mol. Biol. Evol. 17, 540-552. doi: 10.1093/oxfordjournals.molbev.a026334

Chai, B., Wang, H., and Chen, X. (2012). Draft genome sequence of highmelanin-yielding Aeromonas media strain WS. J. Bacteriol. 194, 6693-6694. doi: 10.1128/JB.01807-12

Chan, K. G., Puthucheary, S. D., Chan, X. Y., Yin, W. F., Wong, C. S., Too, W. S., et al. (2011). Quorum sensing in Aeromonas species isolated from patients in Malaysia. Curr. Microbiol. 62, 167-172. doi: 10.1007/s00284-010-9689-z

Chen, H. Q., and Lu, C. P. (1991). Study on the pathogen of epidemic septicemia occurred in cultured cyprinoid fishes in southern China. J. Nanjing Agric. Univ. $14,87-91$.

Chua, P., Har, Z. M., Austin, C. M., Yule, C. M., Dykes, G. A., and Lee, S. M. (2015). Genome sequencing and annotation of Aeromonas sp. Genom. Data 5, 38-39. doi: 10.1016/j.gdata.2015.05.008

Clock, S. A., Planet, P. J., Perez, B. A., and Figurski, D. H. (2008). Outer membrane components of the tad (tight adherence) secreton of Aggregatibacter actinomycetemcomitans. J. Bacteriol. 190, 980-990. doi: 10.1128/Jb.01347-07

Colston, S. M., Fullmer, M. S., Beka, L., Lamy, B., Gogarten, J. P., and Graf, J. (2014). Bioinformatic genome comparisons for taxonomic and phylogenetic assignments using Aeromonas as a test case. mBio 5:e02136. doi: 10.1128/mBio.02136-14

da Silva, B. C., Mourino, J. L. P., Vieira, F. N., Jatoba, A., Seiffert, W. Q., and Martins, M. L. (2012). Haemorrhagic septicaemia in the hybrid surubim (Pseudoplatystoma corruscans $\mathrm{x}$ Pseudoplatystoma fasciatum) caused by Aeromonas hydrophila. Aquac. Res. 43, 908-916. doi: 10.1111/j.13652109.2011.02905.x

Deng, G. C., Jiang, X. Y., Ye, X., Liu, M. Z., Xu, S. Y., Liu, L. H., et al. (2009). Isolation, identification and characterization of Aeromonas hydrophila from hemorrhagic grass carp. China 36, 1170-1177.

Earl, A. M., Onderdonk, A. B., Kirby, J., Ferraro, M. J., Huang, S., Spencer, M., et al. (2015). The Genome Sequence of Aeromonas Hydrophila Strain BWH65. Cambridge, MA: EMBL/GenBank/DDBJ databases.

Erova, T. E., Pillai, L., Fadl, A. A., Sha, J., Wang, S., Galindo, C. L., et al. (2006). DNA adenine methyltransferase influences the virulence of Aeromonas hydrophila. Infect. Immun. 74, 410-424. doi: 10.1128/Iai.74.1.410-4 24.2006

Figueras, M. J., Beaz-Hidalgo, R., Hossain, M. J., and Liles, M. R. (2014). Taxonomic affiliation of new genomes should be verified using average nucleotide identity and multilocus phylogenetic analysis. Genome Announc. 2, e00927-e00914. doi: doi: 10.1128/genomeA.00927-14

Gao, X., Jian, J., Li, W. J., Yang, Y. C., Shen, X. W., Sun, Z. R., et al. (2013). Genomic study of polyhydroxyalkanoates producing Aeromonas hydrophila 4AK4. Appl. Microbiol. Biotechnol. 97, 9099-9109. doi: 10.1007/s00253-013$5189-\mathrm{y}$

Griffin, M. J., Goodwin, A. E., Merry, G. E., Liles, M. R., Williams, M. A., Ware, C., et al. (2013). Rapid quantitative detection of Aeromonas hydrophila strains associated with disease outbreaks in catfish aquaculture. J. Vet. Diagn. Invest. 25, 473-481. doi: 10.1177/1040638713494210

Grim, C. J., Kozlova, E. V., Sha, J., Fitts, E. C., van Lier, C. J., Kirtley, M. L., et al. (2013). Characterization of Aeromonas hydrophila wound pathotypes by comparative genomic and functional analyses of virulence genes. mBio 4, e00064-e00013. doi: 10.1128/mBio.00064-13

Hanson, L., Liles, M. R., Hossain, M. J., Griffin, M., and Hemstreet, W. (2014). "Motile aeromonas septicemia," in Fish Health Section Blue Book 2014 Edition. Bethesda, MD: American Fisheries Society - Fish Health Section. Available online at: http://www.afs-fhs.org/perch/resources/citationguidelines-2014.pdf

Hemstreet, B. (2010). An update on Aeromonas hydrophila from a fish health specialist for summer 2010. Catfish J. 24, 4.

Hemstreet, W. (2015). Aeromonas summary. Fish Farming News: Alabama Fish Farming Center.
Hossain, M. J. (2012). Molecular Interactions between Phage and the Catfish Pathogen Edwardsiella ictaluri and Comparative Genomics of Epidemic Strains of Aeromonas hydrophila. Doctoral Dissertation, Auburn University.

Hossain, M. J., Sun, D., McGarey, D. J., Wrenn, S., Alexander, L. M., Martino, M. E., et al. (2014). An asian origin of virulent Aeromonas hydrophila responsible for disease epidemics in United States-Farmed Catfish. mBio 5, e00848-e00814. doi: $10.1128 / \mathrm{mBio} .00848-14$

Hossain, M. J., Waldbieser, G. C., Sun, D., Capps, N. K., Hemstreet, W. B., Carlisle, K., et al. (2013). Implication of lateral genetic transfer in the emergence of Aeromonas hydrophila isolates of epidemic outbreaks in channel catfish. PLoS ONE 8:e80943. doi: 10.1371/journal.pone.0080943

Jagoda, S. S., Tan, E., Arulkanthan, A., Kinoshita, S., Watabe, S., and Asakawa, S. (2014). Draft genome sequence of aeromonas hydrophila strain Ae34, isolated from a septicemic and Moribund Koi Carp (Cyprinus carpio koi), a freshwater aquarium fish. Genome Announc. 2:e00572-14. doi: 10.1128/genomeA. 00572-14

Kirke, D. F., Swift, S., Lynch, M. J., and Williams, P. (2004). The Aeromonas hydrophila LuxR homologue AhyR regulates the $\mathrm{N}$-acyl homoserine lactone synthase, Ahyl positively and negatively in a growth phase-dependent manner. FEMS Microbiol. Lett. 241, 109-117. doi: 10.1016/j.femsle.2004.10.011

Kitaoka, M., Miyata, S. T., Brooks, T. M., Unterweger, D., and Pukatzki, S. (2011). VasH Is a transcriptional regulator of the type VI secretion system functional in endemic and pandemic Vibrio cholerae. J. Bacteriol. 193, 6471-6482. doi: 10.1128/Jb.05414-11

Leiman, P. G., Basler, M., Ramagopal, U. A., Bonanno, J. B., Sauder, J. M., Pukatzki, S., et al. (2009). Type VI secretion apparatus and phage tail-associated protein complexes share a common evolutionary origin. Proc. Natl. Acad. Sci. U.S.A. 106, 4154-4159. doi: 10.1073/pnas.0813360106

Lenneman, E. M., and Barney, B. M. (2014). Draft genome sequences of the alga-degrading bacteria Aeromonas hydrophila strain AD9 and Pseudomonas pseudoalcaligenes strain AD6. Genome Announc. 2:e00709-14. doi: 10.1128/genomeA.00709-14

Liles, M., Hemstreet, W., Waldbieser, G., Griffin, M., Khoo, L., Bebak, J., et al. (2011). Comparative Genomics of Aeromonas hydrophila Isolates from an Epidemic in Channel Catfish. Auburn, AL: American Society for Microbiology Meeting (1489).

Lim, Y. L., Ee, R., Yin, W. F., and Chan, K. G. (2014). Quorum sensing activity of Aeromonas caviae strain YL12, a bacterium isolated from compost. Sensors 14, 7026-7040. doi: 10.3390/s140407026

Lim, Y. L., Roberts, R. J., Ee, R., Yin, W. F., and Chan, K. G. (2016). Complete genome sequence and methylome analysis of Aeromonas hydrophila strain YL17, isolated from a compost pile. Genome Announc. 4:e0060-16. doi: 10.1128/genomeA.00060-16

Martin, R. L. (1968). Comparison of effects of concentrations of malachite green and acriflavine on fungi associated with diseased fish. Prog. Fish Culturist 30, 153-158.

Martínez-García, P. M., Ramos, C., and Rodriguez-Palenzuela, P. (2015). T346Hunter: a novel web-based tool for the prediction of type III, type iv and type VI secretion systems in bacterial genomes. PLoS ONE 10:e0119317. doi: 10.1371/journal.pone.0119317

Martínez-Murcía, A. J., Saavedra, M. J., Mota, V. R., Maier, T., Stackebrandt, E., and Cousin, S. (2008). Aeromonas aquariorum sp. nov., isolated from aquaria of ornamental fish. Int. J. Syst. Evol. Microbiol. 58(Pt 5), 1169-1175. doi: 10.1099/ijs.0.65352-0

Nielsen, M. E., Høi, L., Schmidt, A. S., Qian, D., Shimada, T., Shen, J. Y., et al. (2001). Is Aeromonas hydrophila the dominant motile Aeromonas species that causes disease outbreaks in aquaculture production in the Zhejiang Province of China? Dis. Aquat. Org. 46, 23-29. doi: 10.3354/Dao046023

Overbeek, R., Olson, R., Pusch, G. D., Olsen, G. J., Davis, J. J., Disz, T., et al. (2014). The SEED and the Rapid Annotation of microbial genomes using Subsystems Technology (RAST). Nucleic Acids Res. 42, D206-D214. doi: 10.1093/nar/gkt1226

Pang, M., Jiang, J., Xie, X., Wu, Y., Dong, Y., Kwok, A. H. Y., et al. (2015). Novel insights into the pathogenicity of epidemic Aeromonas hydrophila ST251 clones from comparative genomics. Sci. Rep. 5:9833. doi: 10.1038/Srep09833

Pang, M. D., Lin, X. Q., Hu, M., Li, J., Lu, C. P., and Liu, Y. J. (2012). Tetrahymena: an alternative model host for evaluating virulence of Aeromonas strains. PLoS ONE 7:e48922. doi: 10.1371/journal.pone.0048922 
Pell, L. G., Kanelis, V., Donaldson, L. W., Howell, P. L., and Davidson, A. R. (2009). The phage lambda major tail protein structure reveals a common evolution for long-tailed phages and the type VI bacterial secretion system. Proc. Natl. Acad. Sci. U.S.A. 106, 4160-4165. doi: 10.1073/pnas.0900044106

Pridgeon, J. W., Zhang, D., and Zhang, L. (2014). Complete genome sequence of a moderately virulent Aeromonas hydrophila strain, pc104A, Isolated from soil of a catfish pond in West Alabama. Genome Announc. 2:e00554-14. doi: 10.1128/genomeA.00554-14

Rasmussen-Ivey, C. R., Figueras, M. J., McGarey, D., and Liles, M. R. (2016). Virulence Factors of Aeromonas hydrophila: in the wake of reclassification. Front. Microbiol. 7:1337. doi: 10.3389/fmicb.2016.01337

Raychaudhuri, S., Saha, A., Ghoshal, T., and Thakur, A. R. (2013). Draft genome sequence of ammonia-producing Aeromonas sp. MDS8 strain MCC2167 from sludge of a dairy effluent treatment plant. Genome Announc. 1:e00710-13. doi: 10.1128/genomeA.00710-13

Rheault, J. G., Vincent, A. T., Trudel, M. V., Brochu, F., Boyle, B., Tanaka, K. H., et al. (2015). Variants of a genomic island in Aeromonas salmonicida subsp salmonicida link isolates with their geographical origins. Vet. Microbiol. 175, 68-76. doi: 10.1016/j.vetmic.2014.11.014

Ribeiro, F. J., Przybylski, D., Yin, S., Sharpe, T., Gnerre, S., Abouelleil, A., et al. (2012). Finished bacterial genomes from shotgun sequence data. Genome Res. 22, 2270-2277. doi: 10.1101/gr.141515.112

Richter, M., and Róssello-Móra, R. (2009). Shifting the genomic gold standard for the prokaryotic species definition. Proc. Natl. Acad. Sci. U.S.A. 106, 19126-19131. doi: 10.1073/pnas.0906412106

Rodriguez, L. M., and Konstantinidis, K. (2014). Kostas Lab $\mid$ ANI Calculator.

Rohmer, L., Hocquet, D., and Miller, S. I. (2011). Are pathogenic bacteria just looking for food? Metabolism and microbial pathogenesis. Trends Microbiol. 19, 341-348. doi: 10.1016/j.tim.2011.04.003

Sandt, C. H., Hopper, J. E., and Hill, C. W. (2002). Activation of prophage eib genes for immunoglobulin-binding proteins by genes from the IbrAB genetic island of Escherichia coli ECOR-9. J. Bacteriol. 184, 3640-3648. doi: 10.1128/Jb.184.13.3640-3648.2002

Seshadri, R., Joseph, S. W., Chopra, A. K., Sha, J., Shaw, J., Graf, J., et al. (2006). Genome sequence of Aeromonas hydrophila ATCC 7966T: jack of all trades. J. Bacteriol. 188, 8272-8282. doi: 10.1128/JB.00621-06

Severi, E., Hood, D. W., and Thomas, G. H. (2007). Sialic acid utilization by bacterial pathogens. Microbiology 153, 2817-2822. doi: 10.1099/mic.0.2007/009480-0

Shrivastava, S., and Mande, S. S. (2008). Identification and functional characterization of gene components of type VI secretion system in bacterial genomes. PLoS ONE 3:e2955. doi:10.1371/journal.pone.0002955

Spataro, N., Farfán, M., Albarral, V., Sanglas, A., Lorén, J. G., Fusté, M. C., et al. (2013). Draft genome sequence of Aeromonas molluscorum strain 848TT, isolated from Bivalve Molluscs. Genome Announc. 1:e00382-13. doi: 10.1128/genomeA.00382-13

Stamatakis, A. (2014). RAxML version 8: a tool for phylogenetic analysis and post-analysis of large phylogenies. Bioinformatics 30, 1312-1313. doi: 10.1093/bioinformatics/btu033

Suarez, G., Sierra, J. C., Sha, J., Wang, S., Erova, T. E., Fadl, A. A., et al. (2008). Molecular characterization of a functional type VI secretion system from a clinical isolate of Aeromonas hydrophila. Microb. Pathog. 44, 344-361. doi: 10.1016/j.micpath.2007.10.005

Tekedar, H. C., Karsi, A., Akgul, A., Kalindamar, S., Waldbieser, G. C., Sonstegard, T., et al. (2015). Complete genome sequence of fish pathogen Aeromonas hydrophila AL06-06. Genome Announc. 3:e00368-15. doi: 10.1128/genomeA.00368-15

Velayudhan, J., Jones, M. A., Barrow, P. A., and Kelly, D. J. (2004). L-serine catabolism via an oxygen-labile L-serine dehydratase is essential for colonization of the avian gut by Campylobacter jejuni. Infect. Immun. 72, 260-268. doi: 10.1128/Iai.72.1.260-268.2004

Wang, M., Luo, Z., Du, H., Xu, S., Ni, B., Zhang, H., et al. (2011). Molecular characterization of a functional type VI secretion system in Salmonella enterica serovar Typhi. Curr. Microbiol. 63, 22-31. doi: 10.1007/s00284-0119935-z

Wheeler, D. L., Church, D. M., Federhen, S., Lash, A. E., Madden, T. L., Pontius, J. U., et al. (2003). Database resources of the national center for biotechnology. Nucleic Acids Res. 31, 28-33. doi: 10.1093/nar/gkg033

Xu, B. H., Yin, Z., Wu, Y. S., and Cai, T. Z. (1993). Studies on the taxonomy of pathogenic bacteria of the bacterial hemorrhagic septicemia in cultured fishes in freshwater. Acta Hydrobiol. Sin. 17, 259-266.

$\mathrm{Xu}$, L., Wang, H., Yang, X., and Lu, L. (2013). Integrated pharmacokinetics/pharmacodynamics parameters-based dosing guidelines of enrofloxacin in grass carp Ctenopharyngodon idella to minimize selection of drug resistance. BMC Vet. Res. 9:126. doi: 10.1186/1746-6148-9-126

Yang, W., Li, N., Li, M., Zhang, D., and An, G. (2016). Complete genome sequence of fish pathogen Aeromonas hydrophila JBN2301. Genome Announc. 4:e01615-15. doi: 10.1128/genomeA.01615-15

Yin, M., Ma, Z., Cai, Z., Lin, G., and Zhou, J. (2015). Genome sequence analysis reveals evidence of quorum-sensing genes present in Aeromonas hydrophila strain KOR1, isolated from a Mangrove Plant (Kandelia obovata). Genome Announc. 3:e01461-15. doi: 10.1128/genomeA.01461-15

Zhang, D., Xu, D.-H., and Shoemaker, C. A. (2016). Experimental induction of motile Aeromonas septicemia in channel catfish by water-borne challenge with virulent Aeromonas hydrophila. Aquac. Rep. 3, 18-23. doi: 10.1016/j.aqrep.2015.11.003

Zhang, Y. L., Arakawa, E., and Leung, K. Y. (2002). Novel Aeromonas hydrophila PPD134/91 genes involved in O-antigen and capsule biosynthesis. Infect. Immun. 70, 2326-2335. doi: 10.1128/IAI.70.5.2326-2335.2002

Conflict of Interest Statement: The authors declare that the research was conducted in the absence of any commercial or financial relationships that could be construed as a potential conflict of interest.

Copyright (C) 2016 Rasmussen-Ivey, Hossain, Odom, Terhune, Hemstreet, Shoemaker, Zhang, Xu, Griffin, Liu, Figueras, Santos, Newton and Liles. This is an open-access article distributed under the terms of the Creative Commons Attribution License (CC BY). The use, distribution or reproduction in other forums is permitted, provided the original author(s) or licensor are credited and that the original publication in this journal is cited, in accordance with accepted academic practice. No use, distribution or reproduction is permitted which does not comply with these terms. 